\title{
GLOBAL PHASE STRUCTURE OF THE RESTRICTED ISOSCELES THREE-BODY PROBLEM WITH POSITIVE ENERGY
}

\author{
KENNETH MEYER AND QIUDONG WANG
}

\begin{abstract}
We study a restricted three-body problem with special symmetries: the restricted isosceles three-body problem. For positive energy the energy manifold is partially compactified by adding boundary manifolds corresponding to infinity and triple collision. We use a new set of coordinates which are a variation on the McGehee coordinates of celestial mechanics. These boundary manifolds are used to study the global phase structure of this gradational system. The orbits are classified by intersection number, that is the number of times the infinitesimal body cross the line of syzygy before escaping to infinity.
\end{abstract}

\section{INTRODUCTION}

McGehee (1974) introduced a new set of coordinates in his study of the collinear three-body problem which had the effect of blowing up the triple collision singularity. By properly rescaling the coordinates and the time, he was able to add a boundary manifold (the collision manifold) at the triple collision singularity. The equation of motion can be extended smoothly onto this boundary and the resulting vector field on it (the so-called fictitious flow) is nontrivial and gradient-like. The study of this fictitious flow has greatly improved the understanding of the behavior of the gravitational system nearby triple collision; see for example Simo (1980), Lacomba and Losco (1980), and Devaney (1980).

Based on both the local information provided by the collision manifold and geometric methods in modern dynamics, much as been done since then. Mather and McGehee (1975) constructed the first example of a noncollision singular solution in the collinear four-body problem, and Xia (1991) proved the Poincaré conjecture that such a noncollision singular solution exists without binary collision. These methods show that heteroclinic phenomena are rather common in gravitational systems (see Moeckel (1981, 1984) and Robinson (1984)), and also, some symbolic subsystems are embedded in grativational systems (see Saari and Xia (1988)).

Since the blowing-up method has stimulated so much progress in the qualitative study of gravitational systems, it is natural to ask if we can do the same at other singularities or at infinity. Boundary manifolds have been constructed at infinity (see Lacomba and Simo (1982)), at singularities of general collision (see Wang (1986)), and for the restricted problem (see ElBialy (1989)). However,

Received by the editors April 16, 1991.

1991 Mathematics Subject Classification. Primary 70F07; Secondary 70F15.

Research of the first author was supported by a grant from the National Science Foundation. 


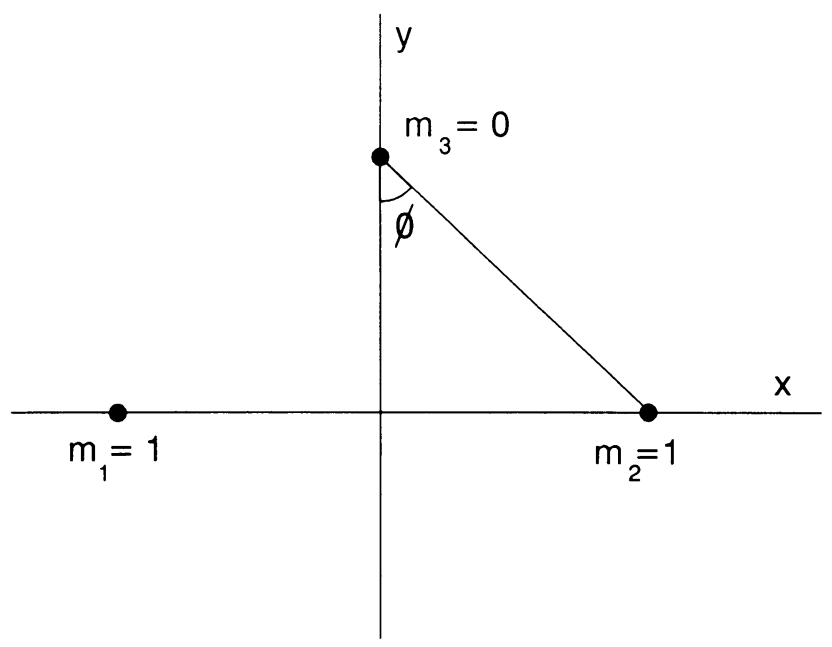

FIGURE 1. The isosceles three-body problem

for a successful generalization, the following requirements should be met: (a) The fictitious flow should be nontrivial and (b) it should provide useful information about the system. We give coordinates to blow up the singularities of our system which meets these requirements.

In this paper we deal with a special gravitational system defined as follows. First, put two gravitational particles with masses $m_{1}=m_{2}=1$ in the $O-X Y$ plane. They are placed on the $X$-axis symmetrically with respect to the $Y$-axis and have only $X$-component initial velocities which are also symmetric with respect to the $Y$-axis. Assume that, as a collinear binary system, $m_{1}, m_{2}$, move apart from each other with positive energy. Now, put another particle with infinitely small mass $m_{3}=0$ on the $Y$-axis and give it only $Y$-component initial velocity. We see $m_{3}$ will always move on the $Y$-axis. The problem is to study the motion of $m_{3}$ in the gravitational field created by $m_{1}$ and $m_{2}$. We call this problem the restricted isosceles three-body problem with positive energy. (See Figure 1.)

We will use the intersection number, which is the number of times the infinitesimal mass $m_{3}$ intersects the $X$-axis, to code solutions. The goal of this paper is to give a global sketch of the variation of this number on phase space.

Our discussion will start from the new blowing-up transformation introduced previously in Wang (1986). The difference between this new transformation and that of McGehee's is that the blowing-up factor was the moment of inertia, $I$, and now is the potential function, $U$. This small modification gives a simple solution to the long-standing global solution problem in celestial mechanics Wang (1991). For a nice description of the global solution problem see Saari (1990).

In this paper, the new transformation allows us to blow up not only the total collision but also other kinds of final evolutions such as the hyperoblic expanding evolution. To be precise, we will get the boundary manifolds for hyperbolic expanding evolution and as well as the collision manifold simultaneously. The first section will be devoted to creating these boundaries and giving a detailed description of the fictitious flows on them. 
According to Marchal and Saari (1976), the only possible final evolution of a solution of our system is hyperoblic expanding. To be precise, if $x_{2}$ denotes the distance between $m_{3}$ and the $X$-axis, then:

$$
x_{2}(t)=A t+B \ln t+C+O\left(t^{-2 / 3}\right),
$$

where either both $A$ and $B$ are nonzero or $A=B=0$. We will refer to the first case $(A, B \neq 0)$ as the hyperbolic solution and to the second case $(A=B=0)$ as the parabolic solution. Using what was introduced in the first section, we will prove the following in the second section of this paper.

(i) The intersection numbers change only near parabolic solution and the change is at most one in the vicinity of any point of the phase space except on the surface $x_{2}=0$. Furthermore, there exist solutions with intersection number $n$ for any given positive integer $n$.

(ii) All of the parabolic solutions form an embedded two-dimensional manifold in the phase space.

In the third section, we will use all the previously obtained conclusions to globally sketch how the intersection number changes in the phase space. It will turn out that the parabolic solutions divide the whole phase space into countably many zones. Each of the zones has an intersection number and these zones are arranged in order of increasing intersection numbers. (See Figure 13.) Thus for this one gradational system the complete description of the global phase space can be given.

\section{THE BOUNDARY MANIFOLDS}

Refer to Figure 1. Let $x_{1}$ denote the distance between the two primaries of mass 1 on the $x$-axis, so they are positioned at $\left( \pm x_{1} / 2,0\right)$ and $x_{2}$ the distance from the infinitesimal particle and the origin so it is located at $\left(0, x_{2}\right)$. The equations for $x_{1}, x_{2}$ are:

$$
\frac{d^{2} x_{1}}{d t^{2}}=-\frac{2}{x_{1}^{2}}, \quad \frac{d^{2} x_{2}}{d t^{2}}=\frac{-16 x_{2}}{\left(x_{1}^{2}+4 x_{2}^{2}\right)^{3 / 2}} .
$$

These are the equations on motion for the restricted isosceles three-body problem. The energy integral for the primaries is

$$
\left(d x_{1} / d t\right)^{2}-4 / x_{1}=h .
$$

We will concentrate on the nature of the motion of this system for positive energy, so $h>0$ throughout this paper. In particular the primaries will escape to infinity. A set of coordinates on the energy manifold is $x_{1}, x_{2}, d x_{2} / d t$. Since $x_{1}>0$ and $x_{2}, d x_{2} / d t$ are arbitrary the energy manifold is topologically $\mathbb{R}^{3}$.

Instead of using the moment of inertia as a scale factor as was done by McGehee (1974) we shall use the potential energy as the scale factor. To this effect let $u^{-1}=\left(4 / x_{1}+h\right)$ and change coordinates from $x_{1}, x_{2}, d x_{1} / d t, d x_{2} / d t$ to $F_{1}, F_{2}, G_{1}, G_{2}$ and time from $t$ to $\tau^{\prime}$ by

$$
\begin{aligned}
F_{1} & =u^{-1} x_{1}, & & F_{2}=u^{-1} x_{2}, \\
G_{1} & =u^{1 / 2}\left(\frac{d x_{1}}{d t}\right), & & G_{2}=u^{1 / 2}\left(\frac{d x_{2}}{d t}\right), \\
d t & =u^{3 / 2} d \tau^{\prime} . & &
\end{aligned}
$$


According to the energy integral, $G_{1}^{2}=1$. We have two different choices of $G_{1}: G_{1}=1$ corresponds to the case $d x_{1} / d t>0$ and $G_{1}=-1$ to $d x_{1} / d t<0$. We will restrict ourselves to the case $G_{1}=1$, so the primaries will tend to infinity as time tends to infinity. Now $F_{1}, F_{2}$, and $G_{2}$ are coordinates on the energy manifold.

With the new time variable $\tau^{\prime}$, the equations for $F_{1}, F_{2}, G_{2}$ are:

$$
\begin{array}{ll}
\frac{d F_{1}}{d \tau^{\prime}}=\left(1-\frac{4}{F_{1}}\right), & \frac{d G_{2}}{d \tau^{\prime}}=\frac{2 G_{2}}{F_{1}^{2}}-\frac{16 F_{2}}{\left(F_{1}^{2}+4 F_{2}^{2}\right)^{3 / 2}}, \\
\frac{d F_{2}}{d \tau^{\prime}}=G_{2}-\frac{4 F_{2}}{F_{1}^{2}}, & \frac{d u}{d \tau^{\prime}}=\frac{4 u}{F_{1}^{2}} .
\end{array}
$$

Now introduce polar coordinates and change the time scale again by

$$
\begin{aligned}
F_{1} & =r \sin \phi, & & F_{2}=(r / 2) \cos \phi, \\
\xi & =1-u h, & d \tau & =(2 \xi)^{-1} d \tau^{\prime},
\end{aligned}
$$

and denote $G=G_{2}$. The angle $\phi=\cot ^{-1}\left(2 F_{2} / F_{1}\right)=\cot ^{-1}\left(2 x_{2} / x_{1}\right)$ and $r^{2}=F_{1}^{2}+4 F_{2}^{2}=\left(x_{1}^{2}+4 x_{2}^{2}\right) / u^{2}$. Since $x_{1}>0$ and $x_{2}$ are arbitrary $0<\phi<\pi$. $G$ is arbitrary and $0<\xi<1$. The new coordinates on the energy manifold are $\phi, G, \xi$ and the equations are

$$
\begin{aligned}
& \frac{d \phi}{d \tau}=\sin \phi\left(\frac{1}{2} \cos \phi-G \sin \phi\right), \\
& \frac{d G}{d \tau}=\frac{\xi\left(G-4 \sin ^{2} \phi \cos \phi\right)}{4}, \\
& \frac{d \xi}{d \tau}=\frac{-\xi(1-\xi)}{2}
\end{aligned}
$$

Notice that these equations naturally extended to $D=[0, \pi] \times(-\infty, \infty) \times$ $[0,1]$ for $(\phi, G, \xi)$. Thus several boundary manifolds have been created by (E2), which we shall discuss in turn.

The boundary manifold $\xi=1$ is the triple ejection manifold. It is a natural generalization of McGehee's collision manifold in this restricted problem. Since there is another choice of $G(G=-1)$, this boundary is only one half of the collision manifold.

Figure 2 is the phase portrait on this boundary. $E$ is the Euler point and $L_{1}, L_{2}$ are the Lagrange points. $E$ is a spiral sink and $L_{1}, L_{2}$ are saddles. $P_{1}, P_{2}$ represent binary ejection of the primaries and $Q_{1}, Q_{2}$ are the intersections of the orbits connecting $P_{1}, L_{1}$ and $P_{2}, L_{2}$ with the dotted line $\phi=\pi / 2$.

The boundary manifold $\xi=0$ is the total expanding manifold $(\xi=0$ means $\left.x_{1}=\infty\right)$. All the points of the curve $\ell: G=\frac{1}{2} \cot \phi$ are rest points as are those on the line $\phi=0$ and $\phi=\pi$. The phase portrait on this boundary is shown in Figure 3.

The boundary manifold $\phi=0$ is the degenerate configuration manifold (since $\phi=0$ means the triangle formed by $\left(m_{1}, m_{2}, m_{3}\right)$ degenerates to a line on the $y$-axis, i.e. $\left.x_{1}=0\right)$. The phase portrait of the fictitious flow is shown in Figure 4 on page 316 .

All the points with $\xi=0$ are rest points. We also have $P_{1}$ in this picture. The boundary manifold $\phi=\pi$ and the fictitious flow on it is exactly the same as that of $\phi=0$. 


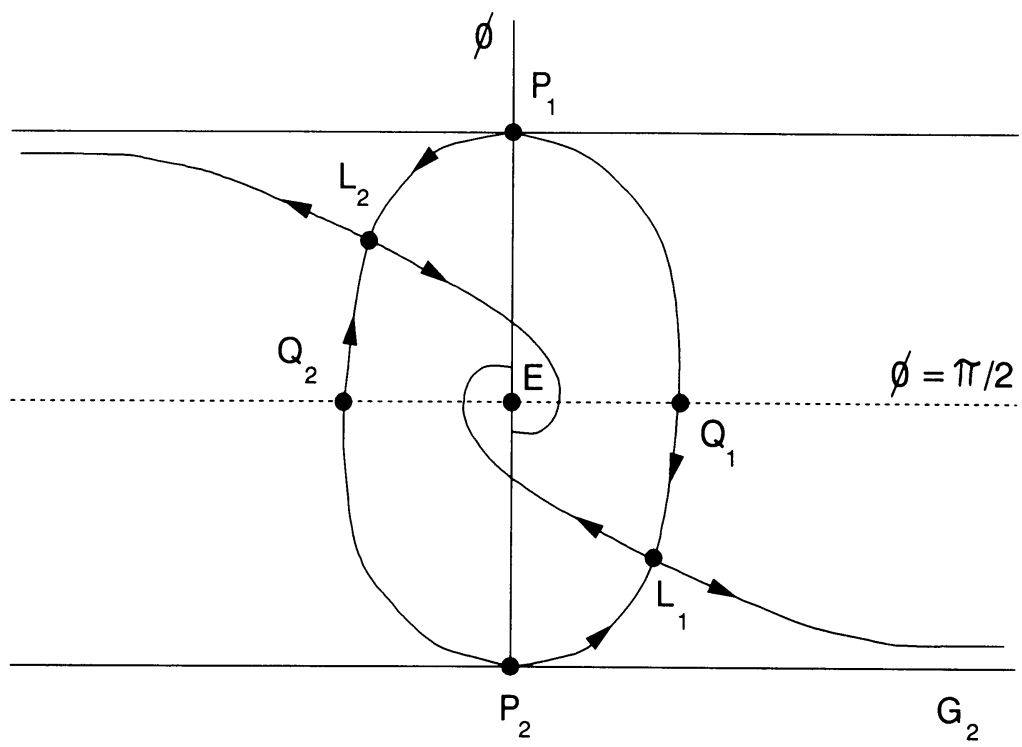

FIGURE 2. The triple ejection manifold $-\xi=1$

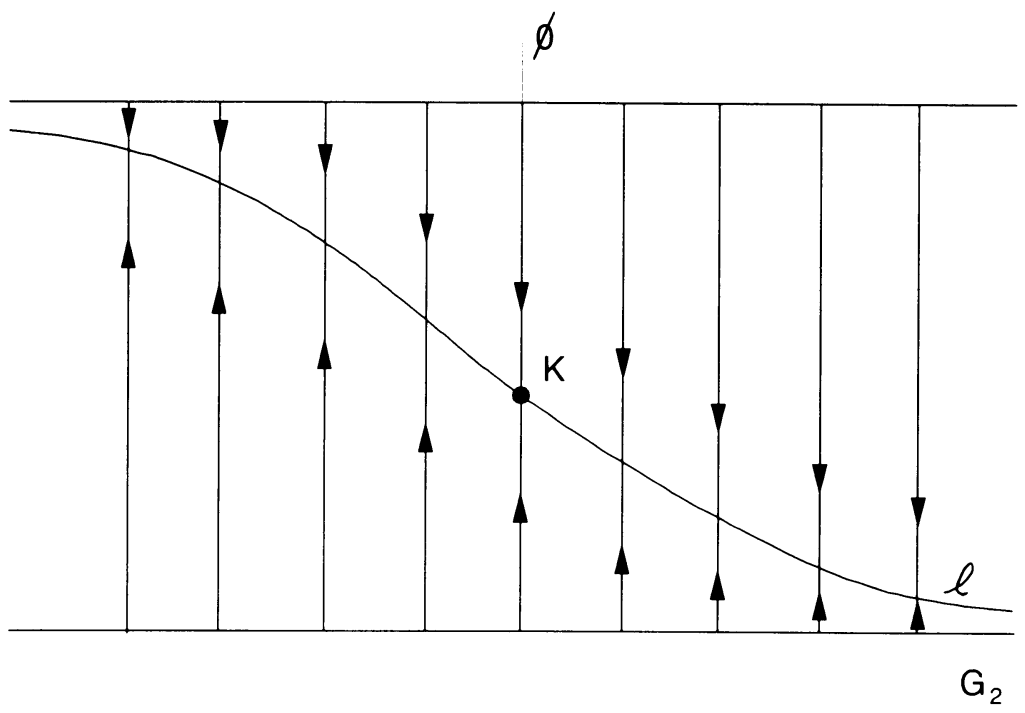

FIGURE 3. The total expanding manifold $-\xi=0$

Figure 5 shows three of the boundaries discussed above, namely the degenerate configuration manifold $\phi=\pi$ in front, the triple ejection manifold, $\xi=1$, on top and the total expanding manifold, $\xi=0$, on the bottom.

We will need some notation for the arguments that follow. Denote by $p$ any point in the phase space $D^{0}=(0, \pi) \times(-\infty, \infty) \times(0,1)$. Denote by $O(\tau, p)$ 


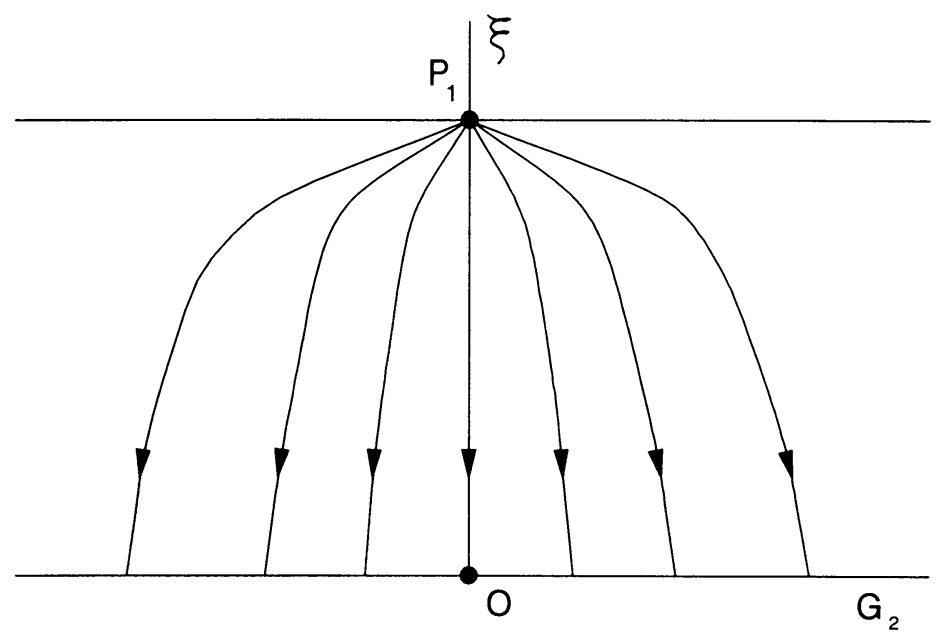

FIGURE 4. Degenerate configuration manifold $-\phi=0$ or $\pi$

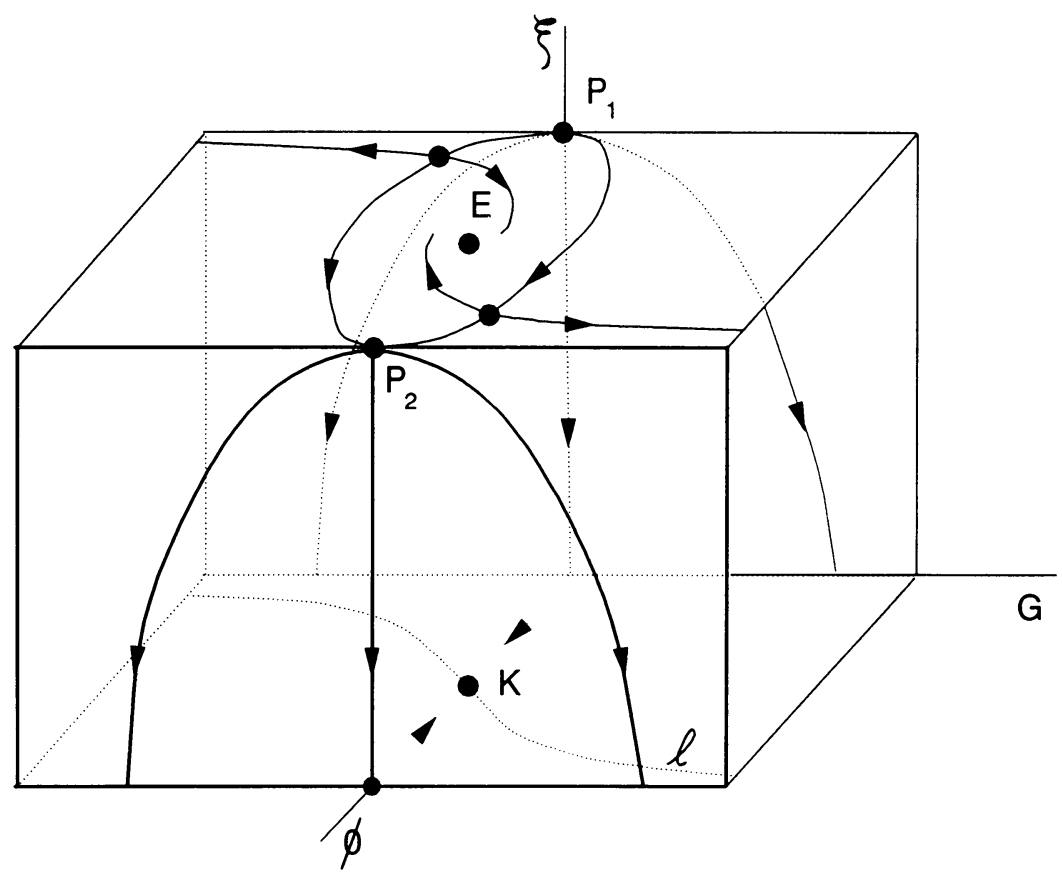

FIGURE 5. Three boundaries

the solution at time $\tau$ which starts from $p$ at $\tau=0$ and

$$
\begin{aligned}
O(\tau, p) & =(\phi(\tau, p), G(\tau, p), \xi(\tau, p)), \\
O(p) & =\{O(\tau, p): 0<\tau<\infty\}, \\
O_{s}(p) & =\{O(\tau, p): 0<\tau<s\}, \\
\omega(p) & =\text { the } \omega \text {-limit set of } O(p), \\
\ell=\{(\phi, G, 0): G & \left.=\frac{1}{2} \cot \phi\right\}, \quad \tilde{\ell}=\ell \backslash(\pi / 2,0,0)=\ell \backslash K,
\end{aligned}
$$




$$
K=(\pi / 2,0,0), \quad S=\{p: \phi(p)=\pi / 2\} .
$$

We also use all the symbols in Figures 2 to 4 .

Definition 1.1. The intersection number of $p$ is defined by

$$
N(p)=\operatorname{Card}\{O(p) \cap S\} .
$$

The intersection number of $p$ is the number of times the orbit through $p$ crosses the $x$-axis in the future.

The following two propositions are simple corollaries of the results in Marchel and Saari (1976) on the final evolutions of gravitational systems. A detailed and independent proof of these two results may be found in Appendix 1.

Proposition 1.1. For any $p, \omega(p)$ is one of the fixed points of $\ell$.

This proposition shows that $\omega$ is a well-defined function on $D^{0}=(0, \pi) \times$ $(-\infty, \infty) \times(0,1)$ into $\ell$.

Proposition 1.2. If $\omega(p) \in \tilde{\ell}$, then

$$
x_{2}(t)=A t+B \ln t+C+O\left(t^{-2 / 3}\right) .
$$

If $\omega(p)=K$ then

$$
x_{2}(t)=C+O\left(t^{-2 / 3}\right), \quad d x_{2} / d t=O\left(t^{-5 / 3}\right),
$$

where $A, B, C$ are constants and $A \neq 0$.

Definition 1.2. If $\omega(p) \in \tilde{\ell}$, we say that $O(p)$ is a hyperbolic solution and $p$ is a hyperbolic point. If $\omega(p)=K$, we say that $O(p)$ is a parabolic solution and $p$ is a parabolic point.

Our first basic lemma establishes the continuity of the function $\omega$ and shows that the intersection number changes only at parabolic points.

Lemma 1.1. The function $\omega: D^{0}=(0, \pi) \times(-\infty, \infty) \times(0,1) \rightarrow \ell$ is continuous. Moreover, if $p_{0} \notin S$ and $\omega\left(p_{0}\right) \neq K$ then there is a neighborhood $U$ of $p_{0}$ where the intersection number $N$ is constant.

Remark. We show that if $\omega\left(p_{0}\right)=a_{0} \in \ell$ then for any $\varepsilon>0$, there is a $\delta>0$ such that when $\left|p-p_{0}\right|<\delta$, we have $\left|\omega(p)-a_{0}\right|<\varepsilon$. In the above $\left|p-p_{0}\right|=\max \left\{\left|f(p)-f\left(p_{0}\right)\right|,\left|G(p)-G\left(p_{0}\right)\right|,\left|x(p)-x\left(p_{0}\right)\right|\right\}$.

Proof. For any given $\varepsilon>0$ and $a_{0}=\left(\phi_{0}, G_{0}, 0\right)$, take the box $B$ as in Figure 6:

$$
\left|\phi-\phi_{0}\right|<\varepsilon_{1}, \quad\left|G-G_{0}\right|<\varepsilon_{2}, \quad|\xi|<\varepsilon_{3} .
$$

Here $\varepsilon_{1}, \varepsilon_{2}, \varepsilon_{3}$ are positive constants less than $\varepsilon$ and

$$
\varepsilon_{2}<1, \quad \varepsilon_{3}<\min \left\{1 / 2,16 \varepsilon_{2} /\left(5\left(G_{0}+5\right)\right\} .\right.
$$

Since $\omega\left(p_{0}\right)=a_{0}$, there is a $\delta>0$ and $U:\left|p-p_{0}\right|<\delta$, such that for any $p \in U$, we have a $\tau_{p}$ with

$$
\left|\phi\left(\tau_{p}, p\right)-\phi_{0}\right|<\varepsilon_{1} / 5, \quad\left|G\left(\tau_{p}, p\right)-G_{0}\right|<\varepsilon_{2} / 5, \quad|\xi|<\varepsilon_{3} .
$$

$O(\tau, p)$ will stay inside $B$ after $\tau_{p}$. Otherwise there will be a $\tau_{2}>\tau_{p}$, such that $O\left(\tau_{2}, p\right)$ is on the boundary of $B$. Referring to Figure 6, we see that the vector field is directed into $B$ on $S_{2}^{ \pm}$where $\phi=\phi_{0} \pm \varepsilon_{1}$. Therefore

$$
G\left(\tau_{2}\right)=G_{0}+\varepsilon_{2} .
$$



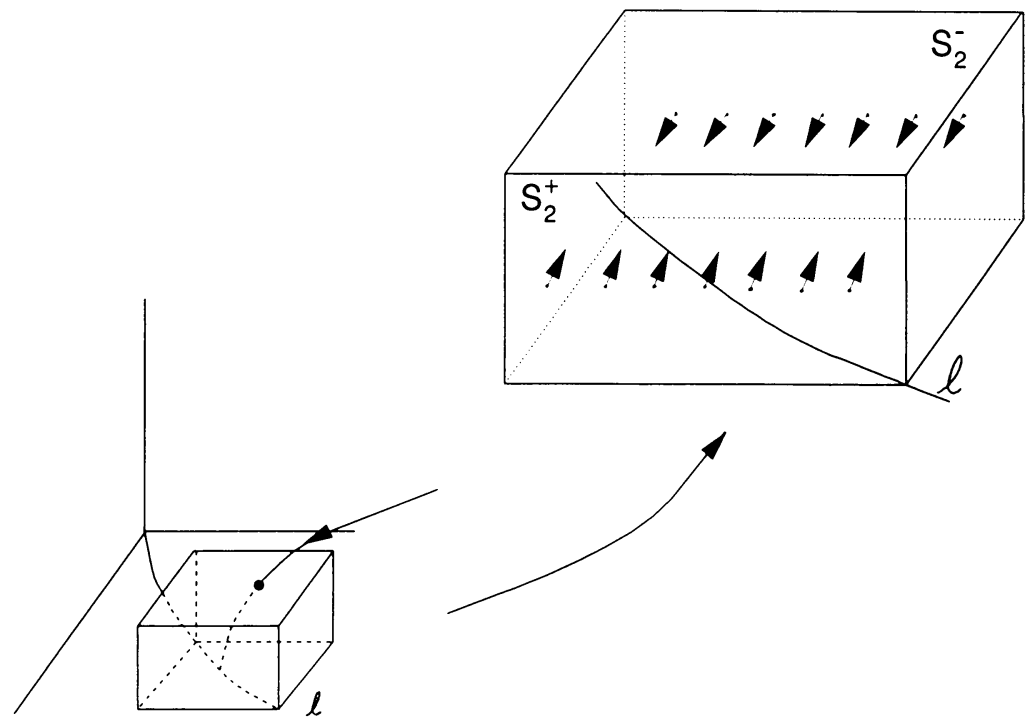

FIGURE 6. The approach to a critical point on $\ell$

Since $\xi$ decrease monotonically, we have

$$
\left|\frac{d G}{d \xi}\right|=\frac{\left|G-4 \sin ^{2} \phi \cos \phi\right|}{2(1-\xi)}<\frac{\left(G_{0}+5\right)}{4} .
$$

Therefore

$$
\left|G\left(\tau_{2}, p\right)-G\left(\tau_{p}, p\right)\right|<\left(G_{0}+5\right) \varepsilon_{3} / 4<4 \varepsilon_{2} / 5
$$

But we also have

$$
\left|G\left(\tau_{2}, p\right)-G\left(\tau_{p}, p\right)\right|=\left|G_{0} \pm \varepsilon_{2}-G\left(\tau_{p}, p\right)\right| \geq \varepsilon_{2}-\left|G\left(\tau_{p}, p\right)-G_{0}\right| \geq 4 \varepsilon_{2} / 5
$$

This is a contradiction.

If $p_{0} \notin S, a_{0} \in \tilde{\ell}=l \backslash K$, we can take $U$ such that $U \cap S=\varnothing$ and $N\left(O_{\tau_{p}}(p)\right)=N\left(p_{0}\right)$.

This lemma shows that the intersection number changes only near parabolic solutions.

\section{THE APPROACH TO THE CRITICAL POINT $K$}

As we have seen $\omega(p) \in l$ for all $p \in D^{0}, \omega(p)=K=(\pi / 2,0,0)$ for a parabolic point $p$, and the intersection number $N(p)$ can only change near a parabolic point. In this section we study the approach to the equilibrium point $K$ in order to obtain information about the manner in which the intersection number changes.

To study the equilibrium point $K$ we introduce another transformation:

$$
\eta=\cos \phi / \xi, \quad \lambda=G / \xi .
$$

The equations for $\eta, \lambda, \xi$ are

$$
\begin{aligned}
& d \eta / d \tau=-\xi \eta / 2+\eta^{3} \xi^{2} / 2+\lambda\left(1-\eta^{2} \xi^{2}\right)^{3 / 2}, \\
& d \lambda / d \tau=\lambda / 2-\xi \lambda / 4-\xi \eta\left(1-\eta^{2} \xi^{2}\right) \\
& d \xi / d \tau=-\xi(1-\xi) / 2
\end{aligned}
$$


Notice that when $\xi=0$, we have a linear flow

$$
d \eta / d \tau=\lambda, \quad d \lambda / d \tau=\lambda / 2 .
$$

All of the points on the line $\lambda=0, \xi=0$ are fixed points. We denote this line by $l^{\prime}$.

Lemma 2.1. $p$ is a parabolic solution if and only if $\omega(p)$ is a fixed point of $l^{\prime}$. Proof. If $\omega(p)$ is a fixed point of $l^{\prime}$, we see that $\cos \phi \rightarrow 0, G \rightarrow 0$. This implies $\omega(p)=(\pi / 2,0,0)$ for $(\phi, G, \xi)$. Therefore, $p$ is a parabolic point.

If $p$ is a parabolic point, then according to the transformations we introduced,

$$
x_{2}=\{2(1-\xi) / h \sin \phi\} \eta .
$$

When $\tau \rightarrow \infty, t \rightarrow \infty$, by Proposition 1.2 we have $x_{2} \rightarrow C$. So $\eta \rightarrow h C / 2=$ $\eta_{0}$ as $\tau \rightarrow \infty$.

Therefore, $\omega(p)$ in $(\eta, \lambda, \xi)$-phase space is $\left(\eta_{0}, 0,0\right) \in l^{\prime}$.

We will prove

Proposition 2.1. For any parabolic point $p_{0} \notin S$, we have a neighborhood $U$ of $p_{0}$ such that for any $p \in U$,

$$
N\left(p_{0}\right) \leq N(p) \leq N\left(p_{0}\right)+1 .
$$

Proof. We shall show in Proposition 2.2 that for any given $\eta_{0}$ there is a unique parabolic orbit with $\eta(t) \rightarrow \eta_{0}$. The $\eta=0, \lambda=0$ line is invariant and consists of the one orbit which has $\eta(t) \rightarrow 0$, so if $\eta(t) \rightarrow 0$ then $\eta(t) \equiv 0$ and $p_{0} \in S$. Therefore, we can assume that $\eta_{0} \neq 0$.

By symmetry we can assume that for this parabolic orbit $x_{2}(t)>0$ and $d x_{2}(t) / d t>0$. Thus we can assume without loss of generality that $\eta_{0}>0$ and $\lambda(t)$ is positive for large $t$. The proof of the proposition depends on a sequence of technical lemmas which will be used again.

For the next three lemmas and the proof of Proposition 2.1 assume that $p_{0} \notin S$ is a parabolic point, $\lim \eta(\tau, p)=\eta_{0}>0$ and $\lambda(t)>0$ for sufficiently large $t$.

Lemma 2.2. For any given $0<\tilde{k}<1 / 24$ and $\varepsilon_{3}<\min \left\{1 / 2, \eta_{0} / 12 M\left(\eta_{0}\right)\right\}$, we have $\varepsilon_{1}, \varepsilon_{2}>0$, so that on $D^{\prime}$ :

$$
\left|\left(G-4 \sin ^{2} \phi \cos \phi\right) /(1-\xi)\right|<\tilde{k} \eta_{0} .
$$

Here $D^{\prime}:|\phi-\pi / 2|<\varepsilon_{1},|G|<\varepsilon_{2},|\xi|<\varepsilon_{3}$, and $M\left(\eta_{0}\right)$ is a constant depending only on $\eta_{0}$. (It will be given explicitly later on in the proof.)

Proof. It is true because $\left.\left(G-4 \sin ^{2} \phi \cos \phi\right)\right|_{(\phi=0, G=0)}=0 ; 1-\xi>1 / 2$.

Lemma 2.3. There is a neighborhood of $p_{0} \notin S$, say, $U\left(p_{0}\right):\left|p-p_{0}\right|<\delta$, such that for any $p \in U\left(p_{0}\right)$, we have $\tau_{p}, O\left(\tau_{p}, p\right) \in D^{\prime}$ and $N\left(p_{0}\right)=N\left(O_{\tau_{p}}(p)\right)$. Furthermore, this solution will stay inside of $D^{\prime}$ after $\tau_{p}$ and $\phi\left(\tau_{p}, p\right)<\pi / 2$.

Remark. In the above $N\left(O_{\tau_{p}}(p)\right)=\operatorname{card}\left\{O_{\tau_{p}}(p) \cap S\right\}$ which is the number of times the orbit through $p_{0}$ intersects $S$ for $0 \leq \tau \leq \tau_{p}$.

Proof. This is an easy corollary of Lemma 1.1. $\phi\left(\tau_{p}, p\right)<\pi / 2$ because $\eta_{0}>$ 0 . 
Lemma 2.4. For any $O(\tau, p)$ in Lemma 3 , assume we have a $\tau_{2}>\tau_{p}$, such that $\left|\lambda\left(\tau_{2}, p\right)\right|>\tilde{k} \eta_{0}$. Then this solution will never meet $G=0$ after $\tau>\tau_{2}$. Actually, it will be a hyperbolic solution.

Proof. If it does meet $G=0$, we have $\tau_{3}>\tau_{2}$ so that $G\left(\tau_{3}, p\right)=0$. (If it is a parabolic solution we have $\tau_{3}=\infty$.) From

$$
d G / d \xi=\left(G-4 \sin ^{2} \phi \cos \phi\right) / 2(1-\xi),
$$

we have

$$
|d G / d \xi| \leq \tilde{k} \eta_{0}
$$

Since $\xi$ decrease monotonically,

$$
\left|G\left(\tau_{3}, p\right)-G\left(\tau_{2}, p\right)\right| \leq \tilde{k} \eta_{0} \xi\left(\tau_{2}, p\right) / 2
$$

But $G\left(\tau_{3}, p\right)=0$. So it follows that

$$
\left|\lambda\left(\tau_{2}, p\right)\right|<\tilde{k} \eta_{0} / 2
$$

This is a contradiction since we assumed $\left|\lambda\left(\tau_{2}, p\right)\right|>\tilde{k} \eta_{0}$.

Now we are ready to prove Proposition 1.1.

According to Lemma 2.3, we have $\widetilde{U} \subset U\left(p_{0}\right)$, a neighborhood of $p_{0}$, such that for any $p \in \widetilde{U}, O\left(\tau_{p}, p\right)$

$$
5 \eta_{0} / 4>\eta\left(\tau_{p}, p\right) \geq 3 \eta_{0} / 4 \text { and } \lambda\left(\tau_{p}, p\right)>0 .
$$

Since $\eta_{0}>0$ and $\lim \eta\left(\tau, p_{0}\right)=\eta_{0}, \widetilde{U}$ exist.

For the motion after $\tau_{p}$, we have two possibilities:

(i) $\lambda>0$ for all $\tau>\tau_{p}$ : Since $\lambda$ is positive $d x_{2} / d \tau$ is positive and the solution will never intersect $S$ again after $\tau_{p}$, and we conclude that $N(p)=$ $N\left(p_{0}\right)$.

(ii) There is a $\tau_{3}$ such that $\lambda\left(\tau_{3}, p\right)=0$ but for $\tau_{p}<\tau<\tau_{3}, \lambda(\tau, p)>0$. Since $\lambda(\tau, p)>0, x_{2}$ will increase monotonically in $\left[\tau_{p}, \tau_{3}\right]$ to $x_{2}\left(\tau_{3}, p\right)$. After that, it will decrease monotonically until the solution meets $S$ at $\tau_{4}>\tau_{3}$. We have $\eta\left(\tau_{4}, p\right)=0$.

Define

$$
\tilde{\tau}=\sup \left\{\tau: \tau \in\left[\tau_{p}, \tau_{4}\right], \eta(\tau, p)=\eta_{0} / 2\right\} .
$$

Obviously $\tilde{\tau} \in\left[\tau_{3}, \tau_{4}\right]$. Now consider the solution in $\left[\tilde{\tau}, \tau_{4}\right]$, for which we also have two possibilities:

(a) We have $\tau \in\left[\tilde{\tau}, \tau_{4}\right],|\lambda(\tau, p)|>\tilde{k} \eta_{0}$ : By Lemma 2.4, we have $N(p)=$ $N\left(p_{0}\right)+1$.

(b) For every $\tau \in\left[\tilde{\tau}, \tau_{4}\right],|\lambda(\tau, p)|<\tilde{k} \eta_{0}$ In this case, on $\left[\tilde{\tau}, \tau_{4}\right]$ we have

$$
|\eta(\tau, p)|<\eta_{0} / 2, \quad|\lambda(\tau, p)|<\tilde{k} \eta_{0}<\eta_{0} / 2 .
$$

Denote $\Phi=\eta-2 \lambda$. From (E3) we have

$$
\begin{aligned}
\frac{d \Phi}{d \tau} & =\xi\left\{-\frac{\eta}{2}+\frac{\eta^{3} \xi}{2}+\frac{\lambda\left[\left(1-\eta^{2} \xi^{2}\right)^{3 / 2}-1\right]}{\xi}+\frac{\lambda}{2}+2 \eta\left(1-\eta^{2} \xi^{2}\right)\right\} \\
& =\xi L(\eta, \lambda, \xi) .
\end{aligned}
$$

But

$$
\left|\left(1-\eta^{2} \xi^{2}\right)^{3 / 2}-1\right|<\left|\left(1-\eta^{2} \xi^{2}\right)^{3}-1\right|=\left|3 \xi^{2} \eta^{2}-3 \eta^{4} \xi^{4}+\eta^{6} \xi^{6}\right| .
$$


So, when $\tau \in\left[\tilde{\tau}, \tau_{4}\right]$, we have

$$
|L(\eta, \lambda, \xi)| \leq M\left(\eta_{0}\right)
$$

where

$$
M\left(\eta_{0}\right)=2 \eta_{0}+\eta_{0}^{2}+\eta_{0}^{3}+\eta_{0}^{4}+\eta_{0}^{6}
$$

Therefore,

$$
|d \Phi / d \xi|<4 M\left(\eta_{0}\right), \quad|\Phi(\tilde{\tau}, p)-\Phi(\tilde{\tau}, p)|<4 M\left(\eta_{0}\right) \xi(\tilde{\tau}, p) .
$$

According to the definitions of $\tilde{\tau}$ and $\tau_{4}$, we have

$$
\Phi(\tilde{\tau}, p)=\eta_{0} / 2-2 \lambda(\tilde{\tau}, p) ; \quad \Phi\left(\tau_{4}, p\right)=-2 \lambda\left(\tau_{4}, p\right) .
$$

So far we have shown

$$
\begin{gathered}
\left|\eta_{0} / 2-2 \lambda(\tilde{\tau}, p)+2 \lambda\left(\tau_{4}, p\right)\right| \leq 4 M\left(\eta_{0}\right) \xi(\tilde{\tau}, p) \\
\eta_{0} / 2-2\left|\lambda(\tilde{\tau}, p)-\lambda\left(\tau_{4}, p\right)\right| \leq 4 M\left(\eta_{0}\right) \xi(\tilde{\tau}, p)
\end{gathered}
$$

Remember that

$$
\left|\lambda(\tilde{\tau}, p)-\lambda\left(\tau_{4}, p\right)\right|<2 \tilde{k} \eta_{0} ; \quad 4 \tilde{k} \eta_{0}>\left(\eta_{0} / 2-4 M\left(\eta_{0}\right) \xi_{3}\right) .
$$

But $\varepsilon_{3}<\eta_{0} / 12 M\left(\eta_{0}\right)$. This implies

$$
4 \tilde{k} \eta_{0}>\eta_{0} / 6, \quad \tilde{k}>1 / 24
$$

which contradict the definition of $\tilde{k}$. The proof of Proposition 2.1 is complete.

To further study the approach to $K$ we will make yet another change of coordinates. Take a parabolic point $p_{0}$. Assume $\eta\left(\tau, p_{0}\right) \rightarrow \eta_{0}$ as $\tau \rightarrow \infty$. Introduce another transformation as follows:

$$
H=\left(\eta-\eta_{0}\right) / \xi^{2 / 3}, \quad L=\lambda / \xi^{2 / 3}, \quad P=\xi^{1 / 3}
$$

The equations for $H, L, P$ are

$$
\begin{aligned}
\frac{d H}{d \tau}= & \frac{H\left(1-P^{3}\right)}{3}+L\left(1-\left(\eta_{0}+H P^{2}\right)^{2} P^{6}\right)^{3 / 2} \\
& -\frac{P \eta_{0}+P^{3} H}{2}+P^{4}\left(P^{2} H+\eta_{0}\right)^{3} \\
\frac{d L}{d \tau}= & \left(\frac{1-P^{3}}{3}+\frac{1}{2}\right) L-P\left(\eta_{0}+H P^{2}\right)\left(1-\left(\eta_{0}+H P^{2}\right) P^{6}\right)-\frac{L P}{4} \\
\frac{d P}{d \tau}= & -\frac{P\left(1-P^{3}\right)}{6}
\end{aligned}
$$

Notice that $(H, L, P)=(0,0,0)$ is an isolated hyperbolic fixed point of (E4) with a two-dimensional unstable manifold, $P=0$, and a one-dimensional stable manifold, $L=H=0$.

Propositon 2.2. For any given $\eta_{0}$, there is one and only one parabolic orbit such that $\eta \rightarrow \eta_{0}$ as $\tau \rightarrow \infty$.

Proof. Recall: (i) According to the relationship between $t$ and $\tau$, we see that when $t \rightarrow \infty, \tau \rightarrow \infty$, and $\xi \sim A t^{-1}$, where $A$ is a constant depending only on $h$; and (ii) a parabolic solution satisfies equation (1.2). 
For any given $\eta_{0}$, we can transform coordinates by (T4) to obtain (E4). Since $(H, L, P)=(0,0,0)$ is a hyperbolic fixed point with a one-dimensional stable manifold, the system has one orbit that tends to $(0,0,0)$. It is a parabolic orbit with $\eta \rightarrow \eta_{0}$ as $\tau \rightarrow \infty$.

From the transformation (T4) again, if $\eta \rightarrow \eta_{0}$ for a solution when $\tau \rightarrow \infty$, and $t \rightarrow \infty$. Clearly $P \rightarrow 0$. But form formula (1.2), we have

$$
H=\left(\eta-\eta_{0}\right) / \xi^{2 / 3} \sim O\left(t^{-2 / 3}\right) t^{2 / 3}=O(1) .
$$

So $H$ is bounded. Similarly, we have

$$
L=\lambda / \xi^{2 / 3} \sim O\left(t^{-5 / 3}\right) t^{5 / 3}=O(1) .
$$

Therefore $L$ is also bounded. Since the orbit is bounded in the future its $\omega$ limit set, $\omega(p)$, is nonempty and the orbit tends to that invariant set. Since $P \rightarrow 0$ the $\omega$-limit set lies in the $P=0$ plane. But the flow on the plane $P=0$ is linear and all orbits other than the origin tend to infinity. Therefore, the $\omega$-limit set is the origin. There is only one such orbit.

Proposition 2.3. For any $p_{0} \notin S$, a parabolic point, and any neighborhood $U$ of $p_{0}$, there is a $p \in U$, such that $N(p)=N\left(p_{0}\right)+1$.

Proof. Without loss of generality, assume $\eta_{0}>0$. We will have $\tau_{0}$ such that $\eta\left(\tau, p_{0}\right)>0, L\left(\tau, p_{0}\right)>0$, and $N\left(O_{\tau_{0}}(p)\right)=N\left(p_{0}\right)$ for this parabolic solution, provided $\tau>\tau_{0}$.

Now take a neighborhood $V$ of $O\left(\tau_{0}, p_{0}\right)$ such that for any $p \in V$ we have $\eta(p)>0, L(p)>0$. For any given neighborhood $U$ of $p_{0}$, we can choose $V$ so small that any point $q \in V$ lies in $U$ and $N_{\tau_{p}}(p)=N\left(p_{0}\right)$ if $q=O\left(\tau_{p}, p\right)$.

Referring to Figure 7 , the unstable manifold of $(0,0,0)$ of (E4) satisfies

$$
d H / d \tau=H / 3+L, \quad d L / d \tau=5 L / 6 .
$$

Take a solution $k$ of $(2.5)$ with initial condition $L<0$ and the segment $s$ as in Figure 7. Consider the backward flow and the time 1 mapping $T$ of the flow. By the $\lambda$-lemma, we have $n$ sufficiently large such that $T^{n}(s) \cap V \neq \varnothing$. Any point of this intersection gives a solution with $N(p)=N\left(p_{0}\right)+1$ since on $s, L<0$.

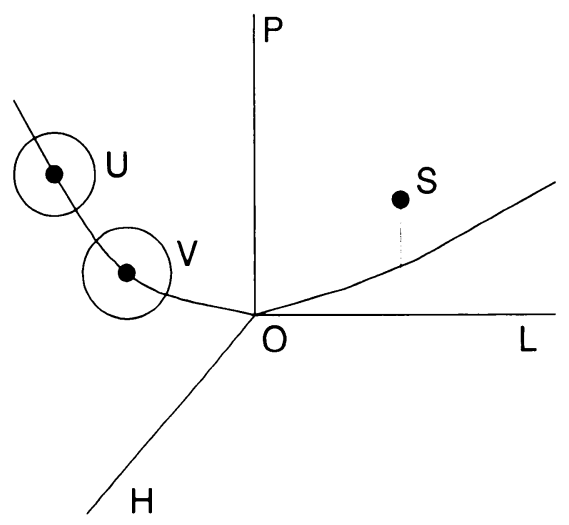

Figure 7 . The approach to $(0,0,0)$ 
One may check that the fixed-points line of (E3) is a normally hyperbolic invariant set. By applying the normal stable manifold theorem (see Hirsch, Pugh, and Shub (1977)), one concludes that all of the parabolic solutions form a two-dimensional immersed manifold in the phase space. Actually we can do much more.

Proposition 2.4. All of the parabolic solutions form a two-dimensional embedded manifold in the phase space.

Proof. For any given $\eta_{0}$, we can use (T4) to conclude (E4). In fact, (E4) is an equation with parameter $\eta_{0}$. Since $(H, L, P)=(0,0,0)$ is a hyperbolic fixed point with one negative eigenvalue, we have one dimensional stable manifold given as

$$
H=H\left(\tau, \eta_{0}\right), \quad L=L\left(\tau, \eta_{0}\right), \quad P=P(\tau) .
$$

Since $P(\tau)$ is monotonically decreasing and independent of $\eta_{0}$, we have $\tau=\tau(P)$. Therefore, we conclude

$$
H=H\left(P, \eta_{0}\right), \quad L=L\left(P, \eta_{0}\right)
$$

for any $\eta_{0} \in(-\infty, \infty), P \in(0,1)$.

Going back to the coordinate $(\eta, \lambda, P)$, we have

$$
\eta=\eta_{0}+P^{2} H\left(P, \eta_{0}\right), \quad \lambda=P^{2} L\left(P, \eta_{0}\right), \quad P=P .
$$

Formula (2.7) defines a map from $D_{2}$ to the phase space $(\eta, \lambda, P)$ :

$$
\begin{aligned}
F: D_{2}= & (-\infty, \infty) \times(0,1) \rightarrow(-\infty, \infty) \times(-\infty, \infty) \times(0,1), \\
& \left(\eta_{0}, P\right) \rightarrow\left(\eta_{0}+H\left(P, \eta_{0}\right) P^{2} ; L\left(P, \eta_{0}\right) P^{2} ; P\right) .
\end{aligned}
$$

The right-hand side of (E4) is a $C^{\infty}$-differentiable function with respect to $\eta_{0}$. Therefore, $F$ is a $C^{\infty}$-mapping. By Proposition 2.2, $p \in F\left(D_{2}\right)$ if and only if $p$ is a parabolic point. We denote $\Lambda=F\left(D_{2}\right)$.

$F$ is one-to-one. Otherwise we assume that $\left(\eta_{1}, P_{1}\right),\left(\eta_{2}, P_{2}\right)$ are such that $F\left(\eta_{1}, P_{1}\right)=F\left(\eta_{2}, P_{2}\right)$. From $(2.7), P_{1}=P_{2}$. So we obtain two parabolic solutions

$$
\begin{array}{lll}
\eta=\eta_{1}+P^{2} H\left(P, \eta_{1}\right), & \lambda=P^{2} L\left(P, \eta_{1}\right), & P=P, \\
\eta=\eta_{2}+P^{2} H\left(P, \eta_{2}\right), & \lambda=P^{2} L\left(P, \eta_{2}\right), & P=P,
\end{array}
$$

starting from the same initial point except $\eta_{1}=\eta_{2}$.

For any $p_{0} \in \Lambda$, we have unique $F^{-1}\left(p_{0}\right) \in D_{2}$. Take any neighborhood $U$ of $F^{-1}\left(p_{0}\right)$ in $D_{2}$, and denote $\Lambda_{U}=F(U)$. In order to prove that $F$ is an embedding, we need to prove that there is a neighborhood $V$ of $p_{0}$ such that $V \cap \Lambda=V \cap \Lambda_{U}$.

Without loss of generality, we assume $F^{-1}\left(p_{0}\right)=\left(\hat{\eta}_{0}, P_{0}\right)$ and $U=\left[\hat{\eta}_{0}-\right.$ $\left.\varepsilon, \hat{\eta}_{0}+\varepsilon\right] \times\left[P_{0}-\varepsilon, P_{0}+\varepsilon\right]=I \times J$. If we have no $V$ such that $V \cap \Lambda=$ $V \cap \Lambda_{U}$, there will be $p_{n} \in D_{2}$ such that (i) $p_{n} \rightarrow p_{0}, p_{n} \notin \Lambda_{U}$ and (ii) $\left\{\eta_{0}\left(F^{-1}\left(p_{n}\right)\right)\right\} \cap I \neq \varnothing$. Here (ii) holds because of Proposition 2.2.

For these $p_{n}$, we have two possibilities:

(i) There is a subsequence $P_{n_{k}}$ such that $\eta_{0}\left(F^{-1}\left(p_{n_{k}}\right)\right) \rightarrow \eta_{0}^{\prime}$ where $\eta_{0}^{\prime} \notin I$.

Since $F$ is one-to-one, $F\left(\eta_{0}^{\prime}, P_{0}\right) \neq F\left(\tilde{\eta}_{0}, P_{0}\right)$. Actually we can take $V$ and a neighborhood $W$ of $F\left(\eta_{0}^{\prime}, P_{0}\right)$ so small that $V \cap W=\varnothing$. According to continuous dependence of the stable manifold on the parameter $\eta_{0}$, we have 


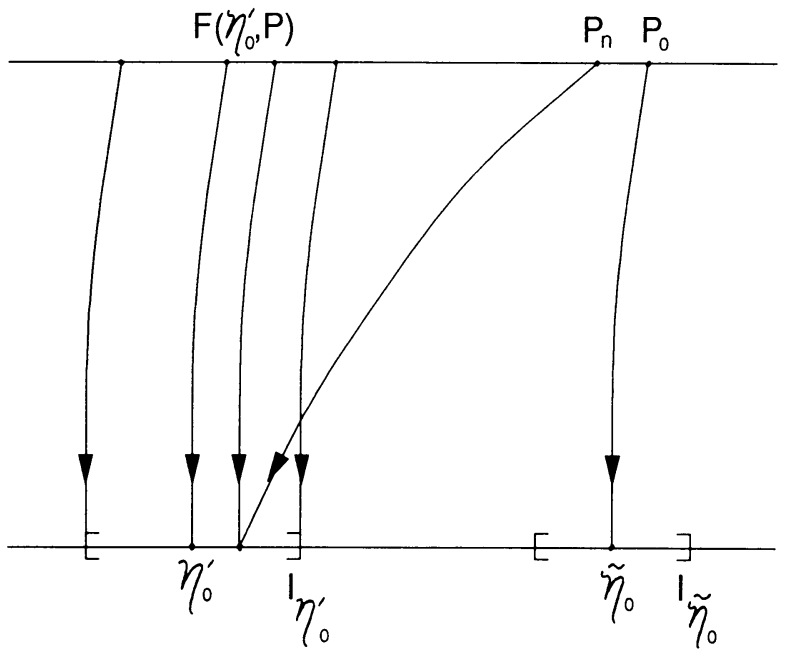

FIGURE 8. Showing uniqueness of limit

an interval including $I^{\prime}=\left(\eta_{0}^{\prime}-\delta, \eta_{0}^{\prime}+\delta\right)$ such that $F\left(I^{\prime} \times\left\{P_{0}\right\}\right) \in W$. Refer to Figure 8 . We have created two different parabolic solutions with same limit value of $\eta_{0}$. This contradicts Proposition 2.2.

(ii) We have $\eta_{0}\left(F^{-1}\left(p_{n}\right)\right) \rightarrow \infty$. Without loss of generality, assume $\eta_{0}\left(F^{-1}\left(p_{n}\right)\right) \rightarrow+\infty$. We are going to show that this case is also impossible.

First we do it in the case $\tilde{\eta}_{0} \neq 0$. Without loss of generality, we assume $\tilde{\eta}_{0}>$ 0 . There will be $p_{n} \in W$ such that $\eta_{0}\left(F^{-1}\left(p_{n}\right)\right)>2 \tilde{\eta}_{0}$ for any neighborhood $W$ of $p_{0}$.

The solution passing through $p_{n}$ is parabolic. Now, take $W$ nearby $p_{0}$ as the $U\left(p_{0}\right)$ in Lemmas 2.2 to 2.4 with $\tilde{k}=1 / 8$. We have $\tau_{p_{n}}$ for $O\left(p_{n}\right)$ so that

$$
3 \tilde{\eta}_{0} / 4<\eta\left(\tau_{p_{n}}, p_{n}\right)<5 \tilde{\eta}_{0} / 4,
$$

and $|\lambda(\tau)|<\tilde{\eta}_{0} / 8$ for all $\tau>\tau_{p}$ since $O\left(p_{n}\right)$ is parabolic.

Now define

$$
\tau^{\prime}=\inf \left\{\tau: \tau>\tau_{p_{n}} ;\left|\eta\left(\tau, p_{n}\right)-\tilde{\eta}_{0}\right|=\tilde{\eta}_{0}\right\}
$$

$\tau^{\prime}$ is well defined and $\left|\eta\left(\tau, p_{n}\right)-\tilde{\eta}_{0}\right|<\tilde{\eta}_{0}$ for any $\tau \in\left(\tau_{p_{n}}, \tau^{\prime}\right)$.

Considering the solution $O\left(p_{n}\right)$ on the interval $\tau \in\left[\tau_{p_{n}}, \tau^{\prime}\right]$. We have shown so far that

$$
|\lambda(\tau)|<\tilde{\eta}_{0} / 8 ; \quad\left|\eta\left(\tau, p_{n}\right)\right|<2 \tilde{\eta}_{0} .
$$

Recall formula (2.2) and the definition $\Phi=\eta-\lambda$ :

$$
\frac{d \Phi}{d \tau}=\xi\left\{-\frac{\eta}{2}+\frac{\eta^{3} \xi}{2}+\frac{\lambda\left[\left(1-\eta^{2} \xi^{2}\right)^{3 / 2}-1\right]}{\xi}+\frac{\lambda}{2}+2 \eta\left(1-\eta^{2} \xi^{2}\right)\right\} .
$$

Substitute (2.9) into it. When $\tau \in\left(\tau_{p}, \tau^{\prime}\right)$, we have

$$
|d \Phi / d \tau| \leq \widetilde{M}\left(\tilde{\eta}_{0}\right),
$$

where $\widetilde{M}\left(\tilde{\eta}_{0}\right)$ is a constant related only to $\tilde{\eta}_{0}$.

Take $\varepsilon_{3}$ in Lemma 2.2 to be $\varepsilon_{3}=\min \left\{1 / 2 ; \tilde{\eta}_{0} / 16 \widetilde{M}\left(\tilde{\eta}_{0}\right)\right\},(2.10)$ yields

$$
\left|\Phi\left(\tau^{\prime}\right)-\Phi\left(\tau_{p_{n}}\right)\right|<\tilde{\eta}_{0} / 16
$$


But

$$
\begin{aligned}
\left|\Phi\left(\tau^{\prime}\right)-\Phi\left(\tau_{p_{n}}\right)\right| & =\left|\eta\left(\tau^{\prime}\right)-\eta\left(\tau_{p_{n}}\right)+2 \lambda\left(\tau_{p_{n}}\right)-2 \lambda\left(\tau^{\prime}\right)\right| \\
& \geq\left|\eta\left(\tau^{\prime}\right)-\tilde{\eta}_{0}+\tilde{\eta}_{0}-\eta\left(\tau_{p_{n}}\right)+2 \lambda\left(\tau_{p_{n}}\right)-2 \lambda\left(\tau^{\prime}\right)\right| \\
& \geq\left|\eta\left(\tau^{\prime}\right)-\tilde{\eta}_{0}\right|-\left|\tilde{\eta}_{0}-\eta\left(\tau_{p_{n}}\right)\right|-2\left|\lambda\left(\tau_{p_{n}}\right)-\lambda\left(\tau^{\prime}\right)\right| \\
& \geq \tilde{\eta}_{0} / 2 .
\end{aligned}
$$

This is a contradiction.

The proof for $\tilde{\eta}_{0}=0$ is almost the same. However, one should change the expression of Lemmas 2.2 to 2.4 properly and repeat the foregoing procedure. Details may be found in Appendix 2.

We need more preparation for the geometric discussion in the next section:

Corollary. For any $n \in Z$, we have $p \in \Lambda$ such that $N(p)=n$.

Proof. Since the Euler point $E$ on the total ejection manifold is a spiral, we can pick a point $q$ such that $N(q)>n$. Take any $q^{\prime}$ in phase space such that (i) $N\left(q^{\prime}\right)=0$. (ii) We have a path $c(s): c(1)=q, c(0)=q^{\prime}$, and $c(s) \cap S=\varnothing$. Such a $q^{\prime}$ exists.

Denote

$$
s_{0}=\sup \{s: N(c(s)) \leq n\} \text {. }
$$

$s_{0}$ is well defined and $s_{0}<1 . c\left(s_{0}\right)$ is a parabolic point since nearby it the intersection number changes. $N\left(c\left(s_{0}\right)\right)=n$ by Proposition 2.1 and the definition of $s_{0}$.

Proposition 2.5. For any given $n \in Z$, we have a neighborhood $U$ of $E$ such that $N(p) \geq n$ provided $p \in U$.

Proof. Recall equation (E2) and denote $k=\cos \phi$. Introduce the polar coordinate system $(R, \vartheta)$ for $(k, G)$ by: $k=R \sin \vartheta, G=R \cos \vartheta$, and let $\xi^{\prime}=1-\xi$.

The equations for $R, \vartheta, \xi^{\prime}$ are

$$
\frac{d \vartheta}{d \tau}=\cos ^{2} \vartheta-\left(\frac{1}{2}+\frac{1-\xi^{\prime}}{4}\right) \cos \vartheta \sin \vartheta+\left(1-\xi^{\prime}\right) \sin ^{2} \vartheta+O(R)
$$

(E4') $\frac{d R^{2}}{d \tau}=2 R^{2}\left(-\frac{\sin ^{2} \vartheta}{2}-\left(1-\xi^{\prime}\right) \sin \vartheta \cos \vartheta+\left(1-\xi^{\prime}\right) \cos ^{2} \vartheta\right)+O\left(R^{3}\right)$

$$
\frac{d \xi^{\prime}}{d \tau}=\frac{\xi^{\prime}\left(1-\xi^{\prime}\right)}{2}
$$

It is easy to see from (E4') that we can find an $\varepsilon>0$ sufficiently small such that, inside the neighborhood $D_{2}^{\prime}$ of $E: 0<R<\varepsilon, 0<\xi^{\prime}<\varepsilon$,

$$
\left|\frac{d \vartheta}{d \tau}\right|>1 / 8, \quad\left|\frac{d R^{2}}{d \tau}\right| \leq 10 R^{2}, \quad\left|\frac{d \xi^{\prime}}{d \tau}\right| \leq 10 \xi^{\prime}
$$

Now for any given $n \in Z$, let $N=e^{80 \pi n}$ and take the neighborhood $U$ of $E$ to be

$$
U: 0 \leq R^{2} \leq \varepsilon / N ; \quad 0 \leq \xi^{\prime} \leq \varepsilon / N
$$

We claim that $N(p) \geq n$ provided $p \in U$.

Consider the solution starting from a given $p \in U . p=\left(R_{0}, \vartheta_{0}\right)$. This solution will say inside as long as $0<\tau<(\ln N) / 10$. Otherwise, we have 
$\hat{\tau}<(\ln N) / 10$ such that $O(\tau, p) \in D_{2}^{\prime}$ when $\tau \in(0, \hat{\tau})$ and either $R(p, \hat{\tau})=\varepsilon$ or $\xi^{\prime}(p, \hat{\tau})=\varepsilon$.

If $R(p, \hat{\tau})=\varepsilon$, we have

$$
R^{2} \leq R_{0}^{2} e^{10 \hat{\tau}}
$$

Therefore,

$$
\hat{\tau} \geq \ln \left(R^{2}(p, \hat{\tau}) / R_{0}^{2}\right) / 10 \geq \ln (N) / 10 .
$$

This is a contradiction. Similarly $\xi^{\prime}(p, \hat{\tau})=\varepsilon$ is impossible.

So the solution will stay inside of $D_{2}^{\prime}$ for $\tau \in(0, \ln (N) / 10)$. Therefore,

$$
\left|\vartheta(T)-\vartheta_{0}\right| \geq T / 8=\ln (N) / 80=\pi n .
$$

This simply means $N(p) \geq n$.

\section{A PoINCARÉ SECTION}

Let $S=\{p:(\phi, G, \xi), \phi=\pi / 2\}$ with coordinates $(G, \xi) \in(-\infty,+\infty) \times$ $(0,1)$. We will denote $S^{+}=(0, \infty) \times(0,1), S^{-}=(-\infty, 0) \times(0,1)$ and $S_{0}=\{0\} \times(0,1), \widetilde{S}=S \backslash S_{0}$. Also, denote $\bar{S}_{c_{1}, c_{2}}=\left[0, c_{1}\right] \times\left[c_{2}, 1\right]$ for any given $c_{1}>0,0<c_{2}<1 . S_{0}$ is the special collinear solution which goes from $E$ on the triple ejection manifold to $K$ on the total expanding manifold. Except at $S_{0}$ one has $d \phi / d \tau>0$ and so $S^{+}$(or $S^{-}$) is a Poincare cross section for the flow. See Figure 9. In this section we shall discuss in detail the intersection of the manifold of parabolic solutions with $S$.

From now on, by a neighborhood $U$ of $p$, we will mean $p \in S$ and $U$ is a neighborhood in $S$ of $p$. For any given $p \in \widetilde{S}, N(p)$ is well defined. $S_{0}$ is actually the parabolic solution with $\eta_{0}=0$.

According to the discussions in the first two sections we have:

(i) For any given hyperbolic point $p_{0} \in \widetilde{S}$, there is a neighborhood $U$ of $p_{0}$ in $\widetilde{S}$ such that $N\left(p_{0}\right)=N(p)$ provided $p \in U$.

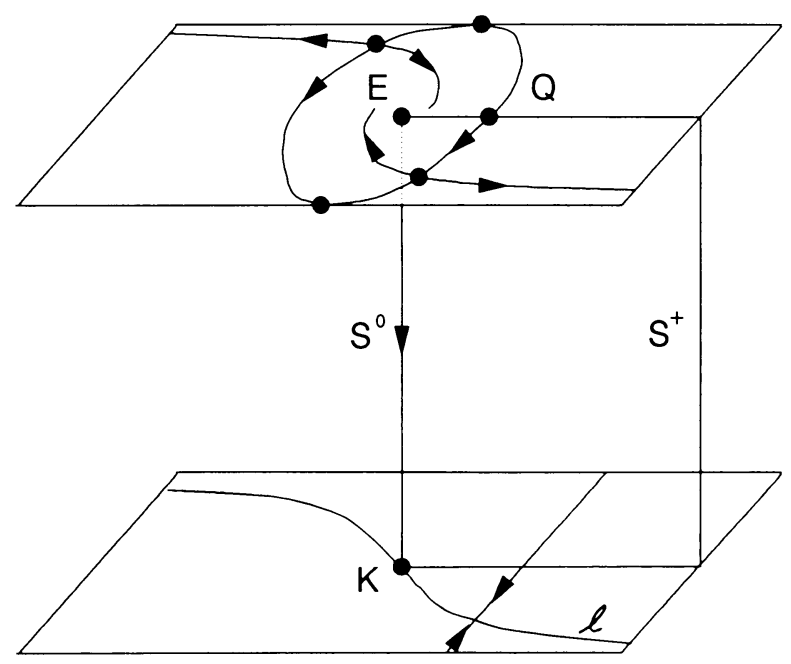

Figure 9. The Poincaré section $S^{+}$ 
(ii) For any given parabolic point $p_{0} \in \widetilde{S}$, we have a neighborhood $U$ of $p_{0}$ in $\widetilde{S}$ such that $N\left(p_{0}\right) \leq N(p) \leq N\left(p_{0}\right)+1$ provided $p \in U$. Furthermore, for any given neighborhood $U$ of $p_{0}$, there are $p_{1}, p_{2} \in U$ such that $N\left(p_{1}\right)=$ $N\left(p_{0}\right), N\left(p_{2}\right)=N\left(p_{0}\right)+1$.

Denote by $\Lambda$ the set of all parabolic solutions. For any $n \in Z^{+}$, define

$$
\Lambda_{n}^{ \pm}=\left\{p: p \in \Lambda \cap S^{ \pm} ; N(p)=n\right\} .
$$

We see that $\Lambda \cap S^{ \pm}=\bigcup_{i=0}^{\infty} \Lambda_{i}^{ \pm}$.

$\Lambda$ is transversal to $S^{ \pm}$since the vector field is transversal to $S^{ \pm}$. So $\Lambda \cap S^{ \pm}$ is a one-dimensional embedded manifold in $S^{ \pm}$. However, it is by no means connected in $S^{ \pm}$.

Now, for any $p \in \Lambda \cap S^{ \pm}$, take the path-connected component $B_{p}$ of $\Lambda \cap S^{ \pm}$ including $p$.

Proposition 3.1. For any $p_{1} \in B_{p_{0}}, N\left(p_{1}\right)=N\left(p_{0}\right)$.

Proof. If we have $p_{1} \in B_{p_{0}}, N\left(p_{1}\right) \neq N\left(p_{0}\right)$. Take the path of $B_{p_{0}}$, say, $c(s):[0,1] \rightarrow B_{p_{0}}$, such that $c(0)=p_{0}, c(1)=p_{1}$. Since $B_{p_{0}}$ is path connected, $c(s)$ exist.

Define $\tilde{s}=\sup \left\{s ; N(c(s))=N\left(p_{0}\right)\right\}$. We see that $0 \leq \tilde{s} \leq 1, c(\tilde{s}) \in \Lambda \cap S^{ \pm}$. According to (ii) in the first part of this section, there is a neighborhood $U$ of $c(\tilde{s})$ such that

$$
N(c(\tilde{s})) \leq N(p) \leq N(c(\tilde{s}))+1
$$

for any $p \in U$. But from the definition of $\tilde{s}$, we have another parabolic point $p^{\prime} \in U$ such that $N\left(p^{\prime}\right) \neq N(c(\tilde{s}))$. Since $N\left(p^{\prime}\right) \geq N(c(\tilde{s}))$, we have $N\left(p^{\prime}\right) \geq N(c(\tilde{s}))+1$.

For $p^{\prime}$, we can take one of its neighborhoods $V$, with $V \subset U$. Again from (ii), there is a $p_{2} \in V$ such that $N\left(p_{2}\right)=N\left(p^{\prime}\right)+1$. This gives $N\left(p_{2}\right) \geq$ $N(c(\tilde{s}))+2$, which is a contradiction since $p_{2} \in U$.

For convenience, we introduce the following definition.

Definition 3.1. Given any $C^{\infty}$-curve in $S, c(s):(-\infty,+\infty) \rightarrow S$,

$$
\omega(c)=\left\{p: \text { there is } s_{n} \rightarrow \infty \text { such that } c\left(s_{n}\right) \rightarrow p\right\}
$$

and

$$
\alpha(c)=\left\{p \text { : there is } s_{n} \rightarrow-\infty \text { such that } c\left(s_{n}\right) \rightarrow p\right\} .
$$

We call $\omega(c)$ and $\alpha(c)$ the $\omega$ - and $\alpha$-limit sets of $c(s)$, respectively.

Definition 3.2. For any connected one-dimensional embedded manifold in $S^{ \pm}$, say, $c(s):(-\infty,+\infty) \rightarrow S$, we call $c(s)$ an upper loop if $\xi(p)=1$ for any $p \in \omega(c) \cup \alpha(c)$. It is a lower loop if $G(p)=0$ for any $p \in \omega(c) \cup \alpha(c)$ and it is a regular branch if for any $p \in \omega(c)$ (respectively $\alpha(c)), \xi(p)=1$ and for any $p \in \alpha(c)$ (respectively $\omega(c)$ ), $G(p)=0$. Referring to Figure 10 , curve 1 is an upper loop, 2 is a lower loop, and 3 a regular curve.

Proposition 3.2. Any connected component $B$ of $\Lambda \cap S^{ \pm}$has no limit point in $S^{ \pm}$.

Proof. Since $\Lambda$ is transversal to $S^{ \pm}, B=B(s)$ is an embedded, path-connected one-dimensional manifold. Since $\lim _{\tau \rightarrow \infty} \eta(\tau, B(s))$ changes monotonically with respect to $s, B(s)$ has no self-intersection so cannot be a closed curve. 


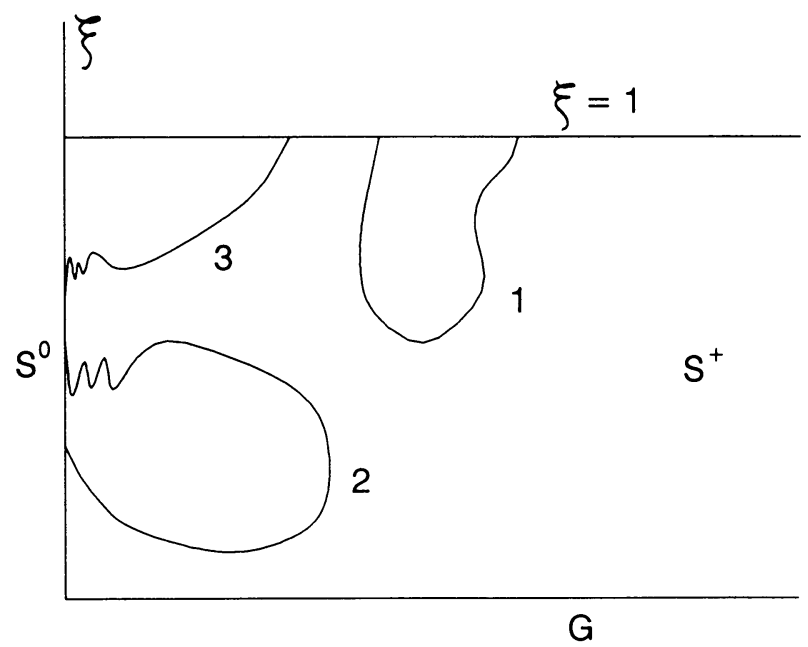

FIGURE 10. Loops and regular branch

Now assume we have a limit point $p$ in $S^{ \pm} . p$ is parabolic (since any hyperbolic point has a neighborhood which contains only hyperoblic points). $p$ is not on $B$. Otherwise there are $s_{n} \rightarrow \infty$ such that $B\left(s_{n}\right) \rightarrow p$. Since $\lim _{n \rightarrow \infty} \lim _{\tau \rightarrow \infty} \eta\left(\tau, B\left(s_{n}\right)\right) \neq \lim _{\tau \rightarrow \infty} \eta(\tau, p)$, it is impossible by the same argument in the proof of Proposition 2.4.

The existence of a path-connected component of $\Lambda \cap S^{ \pm}$passing through $p$, say, $B_{p}$, contradict the fact that $\Lambda \cap S^{ \pm}$is an embedded manifold.

Proposition 3.3. There are $c_{1}, c_{2} ; c_{1}>0,1>c_{2}>0$, such that $\Lambda \cap S^{+} \subset$ $\left[0, c_{1}\right] \times\left[c_{2}, 1\right] ; \Lambda \cap S^{-} \subset\left[-c_{1}, 0\right] \times\left[c_{2}, 1\right]$.

Proof. The first part is to show that there is a $c_{1}>0$ such that any solution starting from $(\pi / 2, G, \xi)$ with $|G|>c_{1}$ will be hyperbolic. We can take $c_{1}=4$. According to the equation

$$
d G / d \tau=\xi\left(G-4 \sin ^{2} \phi \cos \phi\right) / 4,
$$

$G$ will always increase so the solution will be hyperbolic.

There remains the existence of $c_{2}$. Properly expressing it in the original coordinate system $\left(x_{1}, x_{2}, d x_{2} / d t\right)$, we need to show that there is a $c_{2}$ such that no parabolic solution exists starting from $\left(x_{1}(0), 0, d x_{2}(0) / d t\right)$ with $x_{1}(0)>c_{2}$. Certainly we only need to confirm that such a solution with intersection number zero does not exist.

Recall the original equation for $x_{2}$ :

$$
d^{2} x_{2} / d t^{2}=-16 x_{2} /\left(x_{1}^{2}+4 x_{2}^{2}\right)^{3 / 2} \text {. }
$$

If there is a parabolic solution starting from $\left(x_{1}(0), 0, d x_{2}(0) / d t\right)$ with intersection number zero (without loss of generality, assume $d x_{2}(0) / d t>0$ ). We have

$$
x_{2}(t)=\int_{0}^{t}\left[d x_{2}(t) / d t\right] d t \leq\left[d x_{2}(0) / d t\right] t
$$

and

$$
x_{1}^{2}+4 x_{2}^{2} \geq x_{1}^{2} \geq\left(\left[d x_{1}(\infty) / d t\right] t+x_{1}(0)\right)^{2} .
$$


Where $d x_{1}(\infty) / d t$ is a constant depending only on the energy $h$ of the primaries.

Therefore

$$
\begin{aligned}
\frac{d^{2} x_{2}}{d t^{2}} & \geq-\frac{16\left[d x_{2}(0) / d t\right] t}{\left(\left[d x_{1}(\infty) / d t\right] t+x_{1}(0)\right)^{3}} . \\
\frac{d x_{2}(t)}{d t}-\frac{d x_{2}(0)}{d t} & \geq\left\{\int_{0}^{t}\left[\frac{-16 t d t}{\left(\left[d x_{1}(\infty) / d t\right] t+x_{1}(0)\right)^{3}}\right]\right\} \frac{d x_{2}(0)}{d t} \\
& \geq\left\{\frac{-16\left[1 / x_{1}(0)-1 /\left(\left[d x_{1}(\infty) / d t\right] t+x_{1}(0)\right)\right]}{\left[d x_{1}(\infty) / d t\right]^{2}}\right\}\left[\frac{d x_{2}(0)}{d t}\right] .
\end{aligned}
$$

When $t \rightarrow \infty, \lim d x_{2}(t) / d t=0$;

$$
\frac{-d x_{2}(0)}{d t}>\frac{-16}{\left[d x_{1}(\infty) / d t\right]^{2} x_{1}(0)} \frac{d x_{2}(0)}{d t} .
$$

This inequality holds only when

$$
x_{1}(0)<16 /\left[d x_{1}(\infty) / d t\right]^{2} .
$$

Therefore, the choice of $c_{2}=16 /\left[d x_{1}(\infty) / d t\right]^{2}$ will give a contradiction.

Now take any path-connected component $B(s)$ of $\Lambda \cap S^{ \pm}, B(s):(-\infty, \infty) \rightarrow$ $S^{ \pm}$. Without loss of generality, assume that $B(s) \in S^{+}$. Since $\Lambda \cap S^{+} \subset$ $\left[0, c_{1}\right] \times\left[c_{2}, 1\right]=D^{+}$, a compact set, the limit sets $\omega(B(s))$ and $\alpha(B(s))$ are connected subsets of $D^{+}$. By Proposition 3.2, they are on the boundary of $D^{+}$.

Propositon 3.4. For a given $B, \omega(B(s))$ is either a connected subset of $\left(0, c_{1}\right) \times$ $\{1\}$ or of $\{0\} \times\left(c_{2}, 1\right)$.

Proof. By Propositions 2.5 and 3.1, $(0,0)$ cannot be in $\omega(B(s))$.

By Proposition 3.4, $B$ will be one of the three types of curves described in Definition 3.2.

Proposition 3.5. If $\omega(B(s)) \in\left(0, c_{1}\right) \times\{1\}$, it will be either $Q_{1}$ or $Q_{2}$ on the total ejection manifold.

Proof. For any other point $Q$ on $\left(0, c_{1}\right)$, we can find a neighborhood $U$ of $Q$ such that either $N(p)>N(B(s))$ or $p$ is hyperbolic with $N(p)=0$ provided $p \in U$. So $Q$ is not in $\omega(B(s))$.

Now we concentrate on $\Lambda_{0}^{ \pm}$. First of all we have

Proposition 3.6. There is no lower loop for $\Lambda_{0}^{+}$.

Proof. Suppose there is a lower loop $B(s):(-\infty, \infty) \rightarrow S^{+}$. For any given $s$, denote

$$
\lim _{\tau \rightarrow \infty} \eta(B(s), \tau)=\eta_{0}^{B}(s)
$$

We see that

$$
\lim _{s \rightarrow \infty} \eta_{0}^{B}(s)=\eta_{0}^{B}(\infty) \text { and } \lim _{s \rightarrow-\infty} \eta_{0}^{B}(s)=\eta_{0}^{B}(-\infty)
$$

exist since for any $\eta_{0}$, there is only one parabolic solution as with $\eta \rightarrow \eta_{0}$. Also, $\eta_{0}^{B}(\infty) \neq \eta_{0}^{B}(-\infty)$. So at least one of them is nonzero. Without loss of generality, assume $\eta_{0}^{B}(\infty) \neq 0$. 
By Propositions 2.5 and $3.1,(0,0)$ is not in $\omega(B(s))$. Then there is a sequence $s_{n}, s_{n} \rightarrow \infty$, such that

$$
\lim _{n \rightarrow \infty} B\left(s_{n}\right)=\left(0, \xi_{0}\right), \quad \text { where } 0<\xi_{0}<1 .
$$

According to the proof in Appendix 2 and Proposition 2.4, both $\eta_{0}^{B}(\infty)<\infty$ or $\eta_{0}^{B}(\infty)=\infty$ are impossible.

Corollary 3.1. There is no lower loop in $\Lambda_{n}^{ \pm}$for any given $n \in Z$.

Proof. Any lower loop of $\Lambda_{n}^{ \pm}$will create a lower loop for $\Lambda_{0}^{ \pm}$.

Proposition 3.7. There is at most one regular branch for $\Lambda_{0}^{+}$.

Proof. Suppose there are two regular branches, say, $B_{1}(s)$ and $B_{2}(s)$. Assume $\lim _{s \rightarrow \infty} G\left(B_{1}(s)\right)=0, \lim _{s \rightarrow \infty} G\left(B_{2}(s)\right)=0$. Since $\eta_{0}^{B_{1}}(s) \neq \eta_{0}^{B_{2}}(s)$, at least one of these two values is nonzero. But this gives a contradiction similar to that in the proof of Proposition 3.6.

Proposition 3.8. There is one and only one regular branch in $\Lambda_{0}^{+}$.

Proof. We need only establish the existence here. Refer to Figure 11. Take any straight line $G=G_{0}, 0<G_{0}<G\left(Q_{1}\right)$. We claim that it intersects at most finitely many branches of $\Lambda_{0}^{+}$.

If this is not the case, there are infinitely many loops intersecting this line. Then there is a sequence $p_{n}$ on it, with each $p_{n}$ belonging to a different loop branch of $\Lambda_{0}^{+}$. Since we have $c_{1}, c_{2}$, such that $0<c_{1}<\xi\left(p_{n}\right)<c_{2}<1$, $p_{n}$ will have at least one accumulation point inside $S^{+}$, say $p_{0}$. We have $N\left(p_{0}\right)=0$ and $p_{0} \in \Lambda$. The existence of this $p_{0}$ will destroy the embedded manifold structure of $\Lambda_{0}^{+}$.

Now, consider a path, $\pi$, connecting a point with intersection number larger than zero and another point with intersection number zero, We can do it in such a way that this path does not intersect any loop branch of $\Lambda_{0}^{+}$. But it must intersect $\Lambda_{0}^{+}$. Therefore the intersection will be on a regular branch of $\Lambda_{0}^{+}$. The regular branch exists.

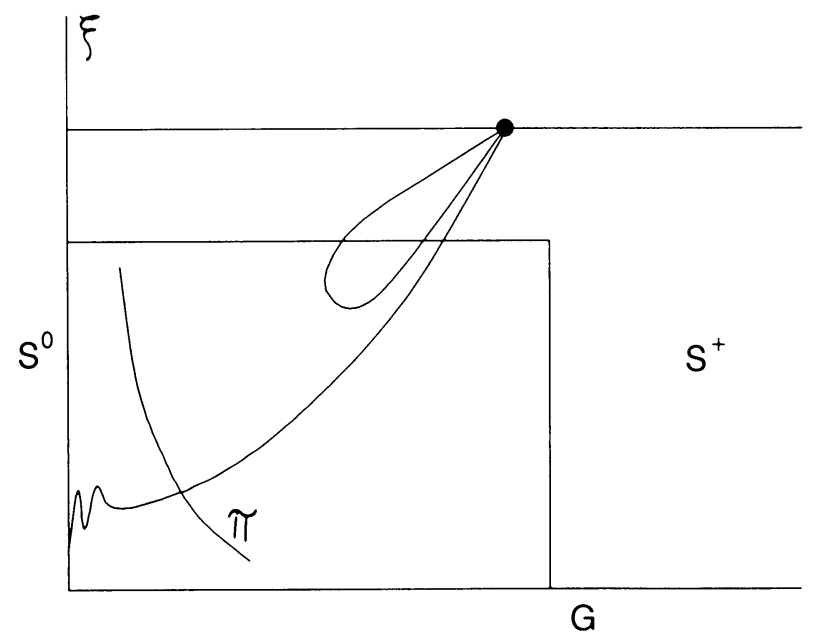

Figure 11. The path $\pi$ in $S^{+}$ 
We will denote this unique regular branch by $R_{0} . R_{0}$ divides $S^{+}$into two parts. Denote again

$D_{0}=\left\{p \in S^{ \pm}:\right.$there is a path connecting $p$ and zero

and there is no point of $\Lambda_{0}^{ \pm}$on this path .

and

$K_{0}=\left\{p \in S^{ \pm}:\right.$there is a path connecting $p$ and $(0,1)$

and there is no point of $\Lambda_{0}^{ \pm}$on this path .

For any $p \in K_{0}, N(p)=0$ and for any $p \in D_{0}, N(p) \geq 1$.

For any $p \in D_{0}$, define

$$
\tau^{*}=\inf \{\tau>0: O(\tau, p) \in S\} .
$$

$\tau^{*}$ exists since $N(p) \geq 1$. See Figure 12 .

Define the Poincaré mapping $F: D_{0}^{ \pm} \rightarrow S$ by $F(p)=O\left(\tau^{*}, p\right)$.

For a given $p \in D_{0}^{ \pm}\left(\right.$or $\left.p \in S^{ \pm}\right), F^{-1}(p)$ might not be well defined. However, for a branch $B$ of $\Lambda_{0}^{ \pm}$, define

$$
F^{-1}(B)=\left\{p: p \in D_{0}^{ \pm}, F(p) \in B\right\} .
$$

Either $F^{-1}(B)=\varnothing$ or $F^{-1}(B)$ is an embedded one-dimensional manifold. Again, $F^{-1}(B)$ is by no means path connected but we have at most countably many path-connected components of $F^{-1}(B)$.

Similarly, for any natural number $n$, define

$$
F^{-n}(B)=\left\{p: p \in D_{0}^{ \pm}, F^{n}(p) \in B\right\} .
$$

Proposition 3.9. If $B$ is a loop, either $F^{-n}(B)=\varnothing$ or $F^{-n}(B)$ consists of only loops.

Proof. For any $n$, if it is not the case, we will have a one-dimensional pathconnected component $c(s):(-\infty, \infty) \rightarrow D_{0}^{ \pm}$, such that $c(s) \in \Lambda_{n}^{ \pm}$, and $c(s)$ is a regular branch of $\Lambda_{n}^{ \pm}$. For $c(s)$, there is a sequence $s_{k} \rightarrow \infty($ or $-\infty)$, such that

$$
\lim _{n \rightarrow \infty} c\left(s_{k}\right)=\left(0, \xi_{0}\right), \quad \text { for some } \xi_{0}>0 .
$$

From the proof of Proposition 4,

$$
\lim _{n \rightarrow \infty} \eta_{0}^{c(s)}\left(s_{k}\right)=0
$$

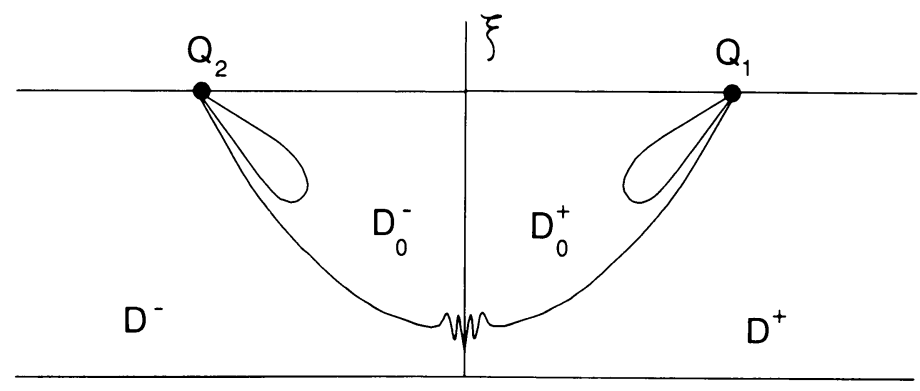

G

FIGURE 12. Regular branches 
Then,

$$
\lim _{n \rightarrow \infty} \eta_{0}^{c(s)}\left(F^{n}\left(s_{k}\right)\right)=0
$$

Therefore, $F^{n}\left(s_{k}\right)$ will lie on a regular branch of $\Lambda_{n}^{ \pm}$. This is a contradiction since they should lie on $B$, a loop of $\Lambda_{0}^{ \pm}$.

So the inverse of a loop is a set of loops.

Proposition 3.10. There is one and only one regular branch in $\Lambda_{n}^{+}\left(\right.$and $\left.\Lambda_{n}^{-}\right)$for any given $n$.

Proof. For the existence, we remark that all of the arguments for the regular branch in $\Lambda_{0}^{+}$apply for the regular branch in $\Lambda_{n}^{+}$. If there is no regular branch in $\Lambda_{n}^{+}$, we can create a contradiction in exactly the same way as in the proof of Proposition 3.8.

For uniqueness, if there are two different regular branches in $\Lambda_{n}^{+}$, we will obtain two different regular branches in $\Lambda_{0}^{+}$by applying the mapping $F^{n}$.

We would like to emphasize that all of the regions and curves we have created in $S^{+}$have their dual in $S^{-}$, symmetrically with respect to the $\xi$-axis of $S$. We will denote the regular branch in $\Lambda_{n}^{ \pm}$by $R_{n}^{ \pm}$.

Proposition 3.11. Assume $(0, \xi) \in \omega\left(R_{n}^{+}\right)\left(\right.$or $\left.\alpha\left(R_{n}^{+}\right)\right)$. For any $\varepsilon>0$. There is an $N$ such that $|1-\xi|<\varepsilon$ for $n>N$.

Proof. If not there is a sequence $p_{n} \in S^{+}$such that $N\left(p_{n}\right) \rightarrow \infty$ and

$$
\lim _{n \rightarrow \infty} p_{n}=(0, \xi) ; \quad|1-\xi|>\varepsilon .
$$

A solution starting from $p_{n}$ will enter the region $|\xi|<c_{2}$ after a fixed period of time $T$ (which is independent of the initial condition $p_{n}$ ). We see that it cannot hit $S$ afterward. Recall the notation in the proof of Proposition 2.5. We can take $n$ sufficiently large so that for $p_{n}$, during $\tau \in[0, T]$, the solution will always satisfy $|O(R)|<1$ in the equation of $d \vartheta / d \tau$.

Therefore, for this solution,

$$
|d \vartheta / d \tau| \leq 5 ; \quad\left|\vartheta\left(T, p_{n}\right)-\vartheta_{0}\right| \leq 5 T
$$

The intersection number will be restricted by $T$ for all $p_{n}$ when $n$ is sufficiently large. This is a contradiction.

This proposition says that the end of the regular branch $R_{n}^{+}$on the $\xi$-axis will move toward $(0,1)$ with increasing $n$.

The following is the global sketch we promised in the beginning of this paper. Refer to Figure 13. Denote the regular branch of $\Lambda_{n}^{ \pm}$as $R_{n}^{ \pm}$. The $R_{0}^{ \pm}$form a region $D_{0}=D_{0}^{+} \cup D_{0}^{-}$with $R_{1}^{ \pm} \in D_{0}$. Again, the $R_{1}^{ \pm}$form a region $D_{1}=$ $D_{1}^{+} \cup D_{1}^{-}$and $R_{2}^{ \pm} \in D_{1}$, and so on in this way we obtain a sequence of regions bounded by $R_{n}^{ \pm}: D \supset D_{0} \supset D_{1} \supset \cdots \supset D_{n} \supset D_{n+1} \cdots$ such that for any $p \in K_{n}: K_{n}=D_{n-1} \backslash D_{n}, N(p)=n$.

Notice that we excluded all of the points inside the loops in $\Lambda \cap S$. So far we have finished our discussion for this special gravitational problem. Evidently the global sketch of the phase structure we have given here is by no means complete. At least two questions are still open. They are:

(a) Do the loops exist in $\Lambda \cap S$ and, if they exist, how does the intersection number change inside the loops?

(b) What is the structure of the limit set of the regular branch on the $\xi$-axis? 


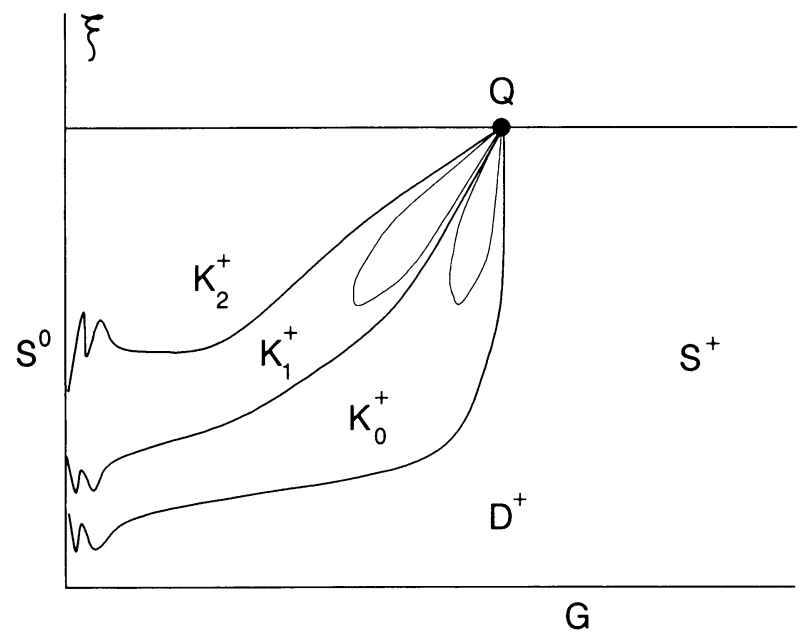

FIGURE 13. The intersection of $S^{+}$with the parabolic solutions

The existence of loops makes the phase portrait very complicated and we hope there are none of them. But frankly we have no idea yet about either their existence or other properties. This seems to be a very essential and difficult question.

\section{APPENDIX 1}

We give independent proofs of Propositions 1.1 and 1.2 here.

Proposition 1.1. For any $p, \omega(p)$ is one of the equilibrium points on $\ell$.

Proof. By (E2), $\xi$ is positive, exponentially and monotonically decreasing. Thus $\int^{+\infty} \xi(s) d s<+\infty$ and $\omega(p)$ is in the total expanding manifold where $\zeta=0$.

Recall Figure 3. We can think of $\ell$ as not only a curve but also as a surface in $(\phi, G, \xi)$ space. $\ell$ divides the phase space into two parts: $d \phi / d t>0$ in one and $d \phi / d t<0$ in the other. Therefore, the line $\phi=0, \phi=\pi$ cannot be the limit points of $O(p)$. Thus $\omega(p)$ must be contained in the curve $\ell$.

We claim that for any given $p, G(p, \tau)$ will be bounded for all $\tau>0$. Otherwise, we have a sufficiently large $\tau_{0}$ with either $G\left(\tau_{0}, p\right)>4$ or $G\left(\tau_{0}, p\right)<-4$. When $G>4$ we have $d G / d \tau>0$ and so $G(\tau, p)>4$ for all $\tau \geq \tau_{0}$. Thus for $\tau \geq \tau_{0}$

$$
\begin{gathered}
\frac{d G}{d \tau} \leq \frac{\xi(t)(G+4)}{4}, \quad \frac{d G}{G+4} \leq \frac{\xi(t)}{4} \\
\ln (G(\tau, p)+4)-\ln \left(G\left(\tau_{0}, p\right)+4\right) \leq \int_{\tau_{0}}^{\tau} \xi(s) d s \leq \int_{\tau_{0}}^{\infty} \xi(s) d s .
\end{gathered}
$$

Which implies $G(\tau, p)$ is bounded. A similar argument holds when $G\left(\tau, p_{0}\right)<$ -4 .

If $|G(\tau, p)|<M$, then $|d G / d \tau|<\xi(\tau)(M+4) / 4$. Since the derivative of $G$ is bounded by an integrable function the $\lim G(p, \tau)$ exist as $\tau \rightarrow \infty$. Denote this value by $G_{0}$. 
Since $\omega(p)$ must lie on the curve $\ell$ and $G(p, \tau) \rightarrow G_{0}$, the $\omega$-limit set is simply the point $(\phi, G, \xi)=\left(\cot ^{-1} 2 G_{0}, G_{0}, 0\right)$.

Proposition 1.2. If $O(\omega(p)) \in \tilde{\ell}=\ell \backslash K$

$$
x_{2}(t)=A t+B \ln t+C+O\left(t^{-\alpha}\right) .
$$

If $\omega(O(p))=K$,

$$
x_{2}(t)=C+O\left(t^{-\alpha}\right) ; \quad d x_{2} / d t=O\left(t^{-\alpha-1}\right)
$$

where $A, B, C$ are constants and $A \neq 0, \alpha$ is any constant with $\alpha<1$.

Proof. From the properties of a two-body problem,

$$
x_{1}(t)=A_{1} t+B_{1} \ln t+C_{1}+O\left(t^{-2 / 3}\right), \quad \frac{d x_{1}}{d t}=A_{1}+\frac{B_{1}}{t}+O\left(t^{-5 / 3}\right),
$$

where $A_{1}=\sqrt{h}>0, B_{1}, C_{1}$ are constants depending only on the energy integral $h$. By Proposition 1.1,

$$
\frac{x_{2}}{x_{1}} \rightarrow G_{0}, \quad \frac{d x_{2} / d t}{d x_{1} / d t} \rightarrow G_{0} .
$$

If $G_{0} \neq 0$, we have

$$
x_{2} \sim G_{0} A_{1} t, \quad d x_{2} / d t \sim G_{0} A_{1} .
$$

Going back to (E0), we have

$$
\frac{d^{2} x_{2}}{d t^{2}}=-\frac{16 x_{2}}{\left(x_{1}^{2}+4 x_{2}^{2}\right)^{3 / 2}}=\frac{B}{t^{2}}+o\left(t^{-2}\right),
$$

where $B$ is a constant related only to $h$ and $G_{0}$.

From (A1),

$$
\frac{d x_{2}}{d t}=\frac{d x_{2}(\infty)}{d t}+\frac{B}{t}+o\left(t^{-1}\right),
$$

where $d x_{2}(\infty) / d t$ is a constant. (In fact, it is $G_{0} A_{1}$.)

Integrating both sides of (A2) again,

$$
x_{2}(t)-x_{2}\left(t_{0}\right)=\left(t-t_{0}\right) \frac{d x_{2}(\infty)}{d t}+B\left(\ln t-\ln t_{0}\right)+O\left(\ln t-\ln t_{0}\right) .
$$

This is actually:

$$
x_{2}(t)=t \frac{d x_{2}(\infty)}{d t}+B \ln t+O(\ln t)
$$

if we take $t_{0}$ fixed and think of (A3) as a function of $t$.

Now, returning to (E0) and substituting (A3), we obtain

$$
\frac{d^{2} x_{2}}{d t^{2}}=\frac{B}{t^{2}}+O\left(\ln \frac{t}{t^{3}}\right)=\frac{B}{t^{3}}+o\left(t^{-3+\alpha}\right)
$$

for any $0<\alpha<1$.

Integrating both sides of (A4),

$$
\frac{d x_{2}(t)}{d t}-\frac{d x_{2}(\infty)}{d t}=\frac{B}{t}+o\left(t^{-2+\alpha}\right)
$$


Let $g(t)=x_{2}(t)-\left[d x_{2}(\infty) / d t\right] t-B \ln t$. For $t$ sufficiently large, we have an $\varepsilon>0$ such that

$$
\left|g\left(t_{1}\right)-g\left(t_{2}\right)\right| \leq \varepsilon\left(t_{1}^{-1+\alpha}-t_{2}^{-1+\alpha}\right) .
$$

Since $\alpha<1$, this means $g(t) \rightarrow g(\infty)$ as $t \rightarrow \infty$. Denote $t_{1}=t$ and let $t_{2} \rightarrow \infty$ in formula (A5); we have

$$
|g(t)-g(\infty)|=o\left(t^{-1+\alpha}\right)
$$

This is exactly

$$
x_{2}(t)=\frac{d x_{2}(\infty)}{d t} t+B \ln t+g(\infty)+O\left(t^{-1+\alpha}\right) .
$$

For the case $G_{0}=0,(\mathrm{~A} 1)-(\mathrm{A} 5)$ are still correct. What we need to do is to set $B=0, d x_{2}(\infty) / d t=0$ in these formulas. We obtain

$$
x_{2}(t)=C+O\left(t^{-1+\alpha}\right) ; \quad d x_{2} / d t=O\left(t^{-2+\alpha}\right) .
$$

Replacing $1-\alpha$ by $\alpha$, we finally obtain the conclusions of Proposition 1.2.

\section{APPENDIX 2}

Here we finish the omitted part in the proof of Proposition 2.4. It is the case $\tilde{\eta}_{0}=0$.

Lemma $2.2^{\prime}$. For any given $0<\tilde{k}<1 / 2$ and $\varepsilon_{3}<1 / 2$, there are $\varepsilon_{1}, \varepsilon_{2}$, such that in $D$,

$$
\left|\left(G-4 \sin ^{2} \phi \cos \phi\right) /(1-\xi)\right|<\tilde{k},
$$

where $D:|\phi-\pi / 2|<\varepsilon_{1},|G|<\varepsilon_{2},|\xi|<\varepsilon_{3}$.

Proof. It is obvious.

Assume $p_{0}$ is a parabolic point; then there is a neighborhood of $p_{0}$, say $U\left(p_{0}\right):\left|p-p_{0}\right|<\delta$, such that for any $p \in U\left(p_{0}\right)$, we have $\tau_{p}, O(\tau, p) \in D_{2}$ for all $\tau>\tau_{p}$ according to Lemma 1.1 .

Lemma $2.4^{\prime}$. For any $p \in U\left(p_{0}\right)$, if there is a $t_{2}>t_{p}$, such that $\left|\lambda\left(\tau_{2}, p\right)\right|>\tilde{k}$, then this solution will be hyperbolic.

Proof. The same argument as in the proof of Lemma 2.4 .

For the case $\tilde{\eta}_{0}=0$ in the proof of Proposition 2.4, assume that we have $p_{n}, \eta_{0}\left(F^{-1}\left(p_{n}\right)\right) \rightarrow \infty$ and $p_{n} \rightarrow p_{0}$. We can take a neighborhood $U\left(p_{0}\right)$ of $p_{0}$ so small that for any $p \in U\left(p_{0}\right)$, there is a $\tau_{p}$ with $\left|\eta\left(\tau_{p}, p\right)\right|<1 / 16$. There will always be infinitely many parabolic points $p_{n}$ inside $U\left(p_{0}\right)$. So we can take $p_{n} \in U\left(p_{0}\right)$ such that $\left|\eta\left(\tau_{p}, p_{n}\right)\right|<1 / 16$. Let

$$
\tau^{\prime}=\inf \left\{\tau: \tau>\tau_{p} ;\left|\eta\left(\tau, p_{n}\right)\right|=1\right\} .
$$

Since $\eta_{0}\left(F^{-1}\left(p_{0}\right)\right) \mid \rightarrow \infty, \tau^{\prime}$ is well defined and $\tau^{\prime}<\infty$.

Since $p_{n}$ is a parabolic point, we have

$$
\left|\lambda\left(\tau, p_{n}\right)\right|<\tilde{k}, \quad\left|\eta\left(\tau, p_{n}\right)\right|<5 / 4
$$

when $\tau \in\left(\tau_{p}, \tau^{\prime}\right)$. Now recall formula $(2.2) ; \Phi=\eta-2 \lambda$ :

$$
\frac{d \Phi}{d \tau}=\xi\left\{-\frac{\eta}{2}+\frac{\eta^{3} \xi}{2}+\frac{\lambda\left[\left(1-\eta^{2} \xi^{2}\right)^{3 / 2}-1\right]}{\xi}+\frac{\lambda}{2}+2 \eta\left(1-\eta^{2} \xi^{2}\right)\right\} .
$$


When $\tau \in\left(\tau_{p}, \tau^{\prime}\right)$, we conclude $|d \Phi / d P|<M_{0}$, where $M_{0}$ is a constant.

Take $\tilde{k}<1 / 16$ and $\varepsilon_{3}=\min \left\{1 / 2,1 / 16 M_{0}\right\}$. It is easy to see that: $\mid \Phi\left(\tau^{\prime}\right)-$ $\Phi\left(\tau_{p}\right) \mid<1 / 16$. But

$$
\begin{aligned}
\left|\Phi\left(\tau^{\prime}\right)-\Phi\left(\tau_{p_{n}}\right)\right| & =\left|\eta\left(\tau^{\prime}\right)-\eta\left(\tau_{p_{n}}\right)+2 \lambda\left(\tau_{p_{n}}\right)-2 \lambda\left(\tau^{\prime}\right)\right| \\
& \geq\left|\eta\left(\tau^{\prime}\right)\right|-\left|\eta\left(\tau_{p_{n}}\right)\right|-2\left|\lambda\left(\tau_{p_{n}}\right)\right|-2\left|\lambda\left(\tau^{\prime}\right)\right| \\
& \geq 11 / 16 .
\end{aligned}
$$

This is a contradiction.

\section{REFERENCES}

R. Devaney, Triple collision in the plannar isosceles three-body problem, Invent. Math. 60 (1980), 249-267.

M. S. ElBialy, Triple collision in the isosceles three-body problem with small mass ratio, J. Appl. Math. Phys. 40 (1989), 645-664.

M. W. Hirsch, C. C. Pugh, and M. Shub, Invariant manifolds, Springer-Verlag, New York, 1977.

E. Lacomba and L. Losco, Triple collision in the isosceles three-body problem, Bull. Amer. Math. Soc. 3 (1980), 489-492.

E. Lacomba and C. Simo, Boundary manifolds for energy surface in celestial mechanics, Celestial Mech. Dynam. Astronom. 28 (1982), 37-48.

R. McGehee, Triple collision in the collinear three-body problems, Invent. Math. 27 (1974), 191-227.

J. N. Mather and R. McGehee, Solution for collinear four-body problem which becomes unbounded in finite time, Lecture Notes in Physics, vol. 3, Springer-Verlag, New York, 1975, pp. 573-597.

R. Moeckel, Heteroclinic phenomena in the isosceles three-body problem, SIAM J. Math. Anal. 15 (1984), 857-876.

, Orbits of the three-body problem which pass infinitely close to triple collision, Amer. J. Math. 103 (1981), 1323-1341.

C. Marchel and D. Saari, On the final evolution of the $n$-body problem, J. Differential Equations 20 (1976), 150-186.

R. Robinson, Homoclinic orbits and oscillation for the planar three-body problem, J. Differential Equations 52 (1984), 356-377.

D. Saari, $A$ visit to the Newtonian $N$-body problem via elementary complex variables, Amer. Math. Monthly 97 (1990), 105-119.

D. Saari and Z.-H. Xia, Oscillatory and super-hyperbolic solutions in Newtonian system, J. Differential Equations 82 (1988), 342-355.

C. Simo, Analysis of triple collision in the isosceles three-body problem, Classical Mechanics and Dynamical Systems, Marcel Dekker, New York, 1980.

Q-D. Wang, Qualitative study of n-body problem: Unitized momentum transformation and its application, Space Dynamics and Celestial Mechanics (K. B. Bhatnagar, ed.), Reidel, 1986, pp. 61-69.

_, The global solution of n-body problem, Celestial Mech. 50 (1991), 73-81.

Z-H. Xia, The existence of non-collision singularity in Newtonian gravitational system, preprint.

InStitute for Dynamics, Department of Mathematical Sciences, University of Cincinnati, Cincinnati, Ohio 45221-0025 